\title{
A Positive Energy Dynamics and Scattering Theory for Directly Interacting Relativistic Particles
}

\author{
S. N. M. RUIJSENAARS* \\ Joseph Henry Laboratories of Physics, Princeton University, Princeton, New Jersey 08544
}

Received October 12, 1979

Starting from the tensor product of $N$ irreducible positive energy representations of the Poincaré group describing $N$ frec relativistic particlcs with arbitrary spins and positive masses, we construct an interacting positive energy representation by modifying the total 4-momentum operator. We first make a transformation to a Hilbert space on which the free total 4-momentum operator equals the product of a dimensionless center-of-mass 4-vector $\left(\left(|\mathbf{k}|^{2}+1\right)^{1 / 2}, \mathbf{k}\right)$ and a free "reduced Hamiltonian" $H_{r}^{0}$, which is a positive operator acting only on internal variables, and then replace $H_{r}{ }^{0}$ by an interacting reduced Hamiltonian $H_{r}=H_{r}^{0}+V$, where $V$ commutes with the Lorentz group and is such that $H_{r}$ is a positive operator. The resulting product form is shown to imply that the wave operators intertwine the free and interacting representations so that the $S$-operator is Lorentz invariant. From a physical point of view the scheme is related to the framework first introduced by Bakamjian and Thomas, in which the Hamiltonian and boost generators are modified, but the above procedure makes a mathematically rigorous discussion much simpler. In the spin-zero case we introduce a natural generalization of the pair potentials of nonrelativistic $\mathrm{N}$-particle Schrödinger theory to the present relativistic setting, study its scattering theory, and point out some problems that do not have analogs at the nonrelativistic level. In the spin- $\frac{1}{2}$ case we propose, inspired by the Dirac equation, explicit reduced Hamiltonians to describe atomic energy levels and present arguments making plausible that their eigenvalues are in closer agreement with the experimental data than their nonrelativistic counterparts. We also consider extensions to arbitrary spin and, in the spin- $\frac{1}{2}$ case, coupling of a quantized radiation field. In view of eventual applications to "completely integrable" one-dimensional field theories the case of one space dimension is studied as well, both in quantum mechanics and in classical mechanics.

\section{INTRODUCTION}

In recent years it has been argued that a number of one-dimensional relativistic quantum field theories has the property that no particle production occurs, in the sense that only $n$-particle to $n$-particle scattering amplitudes are nonzero. Moreover, the set of incoming momenta equals the set of outgoing momenta and the amplitudes factorize into products of two-particle amplitudes, corresponding to a picture in which each incoming particle suffers independent collisions with all the other ones. The simplest examples of this behavior are the Federbush model [1-3] and the field theory

\footnotetext{
* Supported in part by NSF Grant PHY 7823952.
} 
associated to the two-dimensional Ising model in the scaling limit [4]. In these theories the amplitudes are energy-independent phase factors. Other examples are the massive Thirring model/sine-Gordon theory and the nonlinear $\sigma$-model. Explicit energy-dependent expressions for the amplitudes have been proposed in Refs. [5, 6], and have been verified in low orders of perturbation theory. (A review of this and related work can be found in Ref. [7].) The occurrence of these surprising properties is intimately linked to the existence of infinitely many conservation laws, and can be regarded as the quantum analog of the soliton character of some of these models regarded as classical field theories: a classical solution containing $N$ solitons in the distant past will also contain $N$ solitons with the same momenta in the distant future, the only effect of the interaction being a shift in position that is the sum of the shifts that would occur in independent two-soliton collisions [8-11].

The first field theory where this behavior was established at the quantum level is a nonrelativistic one, viz., the quantized nonlinear Schrödinger equation, or equivalently, the $N$-body Schrödinger equation with $\delta$-function pair potentials [12-14]. (The soliton character of the classical theory was established later in this case $[15,16]$.) In this theory not only the free quantum field Hamiltonian but also the interaction Hamiltonian are manifestly particle-number preserving in the sense that they leave the $N$-particle sector in Fock space invariant. The same situation obtains for the massive Thirring model on the unphysical negative energy sector $[17,18]$. For the positive energy massive Thirring model the situation is less clear. Of course the formal interaction Hamiltonian is not particle-number preserving on the free Fock space, but as is well-known, the theory cannot be sensibly formulated in this space.

However, if one assumes that this model and other "completely integrable" field theories could be rigorously constructed as field theories satisfying the Wightman axioms [19], that the Haag-Ruelle theory [20-22] would be applicable, and that the resulting $S$-operators would be equal to the $S$-operators proposed for these theories, then the absence of particle production would imply that the incoming and outgoing $N$-particle sector (as defined in the Haag-Ruelle theory) are equal, so that there is no ambiguity in referring to "the $N$-particle sector." Since the $N$-particle sector is left invariant by the representation of the Poincare group, it is in particular left invariant by the Hamiltonian. (In the case of the sine-Gordon theory a rigorous construction has been performed [23-24], but it seems very hard to verify that the $S$-operator is the one proposed by Zamolodchikov [5].)

Since the rigorous construction of relativistic quantum field theories is very difficult and implicit, even in the superrenormalizable case (cf. Refs. [25, 26]), one may ask the question whether one can construct a relativistic dynamics of directly interacting particles (which would be manifestly particle-number preserving) leading to the $S$-matrices of the above-mentioned class of renormalizable field theories without particle production. (At the classical level a similar question can be asked: is it possible to construct $N$-particle Hamiltonian systems that are relativistically invariant and whose scattering behavior mimics that of the $N$-soliton solutions to the classical partial differential equations (i.e., the same phase shifts occur)? Compare in this connection the intriguing relations between completely integrable nonrelativistic 
$N$-particle systems and the Korteweg-deVries equation $[27,28]$.) The point of this is the following: One can formulate the Haag-Ruelle theory in terms of wave operators $W_{ \pm}: \mathscr{H}_{0} \rightarrow \mathscr{H}$ that intertwine the free representation of the Poincare group, $U_{0}(a, \Lambda)$, on the Fock space $\mathscr{H}_{0}$ of free asymptotic particles, and the interacting representation $U(a, A)$ on the physical Hilbert space $\mathscr{H}$ [22]. The $S$-operator $S_{0} \equiv W_{+}^{-1} W_{-}$on $\mathscr{H}_{0}$ has the same matrix elements as the $S$-operator on $\mathscr{H}$ defined in the usual way. Let us now assume one could construct a theory $\left\{\mathscr{H}^{\prime}, U^{\prime}(a, \Lambda)\right\}$ with wave operators $W_{ \pm}^{\prime}: \mathscr{H}_{0} \rightarrow \mathscr{H}^{\prime}$ intertwining $U_{0}(a, A)$ and $U^{\prime}(a, A)$. Then the operators $E_{ \pm}: \mathscr{H} \rightarrow \mathscr{H}^{\prime}$ defined by $E_{ \pm}=W_{ \pm}^{\prime} W_{ \pm}^{-1}$ intertwine $U(a, A)$ and $U^{\prime}(a, \Lambda)$, and one can transport the field(s) $\phi$ on $\mathscr{H}$ to $\mathscr{H}^{\prime}$ by setting $\phi_{ \pm}^{\prime} \equiv E_{ \pm} \phi E_{ \pm}^{-1}$. The two theories will be equivalent if $\phi_{+}^{\prime}=\phi_{-}^{\prime}$. It is easy to see that this is the case if and only if the $S$-operator $S_{0}^{\prime}=$ $W_{+}^{\prime-1} W_{-}^{\prime}$ equals $S_{0}$.

Summarizing, this argument shows that any relativistically invariant theory $\left\{\mathscr{H}^{\prime}, U^{\prime}(a, A)\right\}$ of directly interacting particles that leads to the known $S$-matrix of a field theory without particle production through wave operators intertwining the tree representation corresponding to the field theory and the representation $U^{\prime}(a, \Lambda)$ on $\mathscr{H}^{\prime}$ will be really the same in the sense of unitary equivalence effected by the wave operators. It seems to us that constructing such a theory may be much easier than constructing the full field theory, and that the former construction might facilitate the latter.

The simplest relativistic theory of directly interacting particles which one might consider would be to add to the free $N$-particle Dirac Hamiltonian an interaction term (pair potentials, e.g.), and to change the other generators of the free representation of the Poincaré group so as to obtain a new representation in which the new Hamiltonian is the generator of time translations. However, existence of the wave operators would then imply that this Hamiltonian has unphysical negative energies in its spectrum like the free one, precluding unitary equivalence to a positive energy theory. (Moreover, relativistic kinematics in one space dimension shows that no reflection can occur between a positive and a negative energy particle, while particle/antiparticle reflection does occur in the massive Thirring model; cf. in this connection Refs. [17, 29].)

The next possibility that suggests itself to avoid negative energies is to modify the Hamiltonian and other free generators of a theory which is the tensor product of $N$ irreducible positive energy representations of the Poincaré group so as to obtain a new "interacting" positive energy representation. In this paper we present a scheme which performs this objective, and is, morcover, such that the wave operators intertwine the free and interacting representations (if they exist). Correspondingly, the $S$-operator is Lorentz invariant, i.e., commutes with the free representation. Although this scheme arose in connection with one-dimensional field theories, it can be easily applied to $N$ particles with arbitrary spins and positive masses in three space dimensions, and therefore seems of independent interest. Consequently, we propose to discuss the scheme in this paper, while its relation to completely integrable field theories will be considered elsewhere.

After the main features of the scheme were established a conversation with Wightman made the author aware of a rather large body of related work concerning 
relativistic particle dynamics in three dimensions. The framework developed in the literature consists in modifying the free Hamiltonian and boost generators while keeping the other generators unchanged (an "instant" form of dynamics in Dirac's terminology [30]), while we modify the momentum 4-vector and leave the Lorentz group unchanged (a "point" form of dynamics, also studied in Ref. [31]). We mention Refs. [32, 33], where this framework was first introduced, Refs. [34, 35], where its scattering theory was studied, and the recent reference [36] where many other references can be found. The fact that the total 4-momentum can be written as the product of a center-of-mass 4-vector and an internal "reduced Hamiltonian" (which does not seem to have been realized or emphasized before) makes the framework discussed in this paper considerably simpler and more natural in our opinion. Moreover, certain mathematical pathologies can be easily discarded as will be discussed below.

\subsection{The Main Features of the Scheme}

Before outlining the rest of the paper we shall first describe the main features of the scheme, assuming for simplicity that the $N$ relativistic particles are distinguishable and have masses $m_{i}>0(i=1, \ldots, N)$, and have zero spin and no internal degrees of freedom. Particles with nonzero spin will be considered in Section 5 , while the imposition of statistics or the addition of internal degrees of freedom does not pose any problems of principle. Presumably one could also cover the case of one or more particles being massless but we shall not pursue this.

Under our assumptions the state space of the particles in an $n+1$ - dimensional space-time can be taken to be $\mathscr{H}=L^{2}\left(R^{n N} ; d \mathbf{p}_{1} \cdots d \mathbf{p}_{N}\right)$ where the $\mathbf{p}_{i}$ are the $n$ momenta of the particles. In the main text $n$ will be 1 or 3 . (For $n=1$ we shall actually use the more convenient particle rapidities.) This space carries the free representation of the Poincaré group, denoted by $U_{f}(a, \Lambda)$ (cf. the Appendix for some details for $n=3$ ). The free Hamiltonian and $n$-momentum operators are the generators of time resp. space translations and are given by

$$
\begin{aligned}
& H_{f}=\sum_{i=1}^{N} E_{p_{i}}, \\
& \mathbf{P}_{f}=\sum_{i=1}^{N} \mathbf{p}_{i},
\end{aligned}
$$

where

$$
E_{p_{i}} \equiv\left(\left|\mathbf{p}_{i}\right|^{2}+m_{i}^{2}\right)^{1 / 2}
$$

As will be detailed in the next sections one can now make a transformation to a dimensionless center-of-mass variable $\mathbf{k}$ and $N-1$ momenta describing the internal motion of the particles. There is then a corresponding unitary mapping $I$ of $\mathscr{H}$ onto a Hilbert space $\overline{\mathscr{H}} \simeq \mathscr{H}_{\mathrm{CM}} \otimes \tilde{\mathscr{H}}$, where $\mathscr{H}_{\mathrm{CM}}=L^{2}\left(R^{n} ; d \mathbf{k}\right)$ and $\tilde{\mathscr{H}}$ is the Hilbert space corresponding to the internal momenta. It will be convenient to regard $\overline{\mathscr{H}}$ as a 
constant fiber direct integral with fiber $\tilde{\mathscr{H}}$ (for a concise exposition of the main properties of such integrals see Ref. [37, pp. 280-286]). The transformation is such that on $\overline{\mathscr{H}}$ the free $n+1$-momentum

$$
P_{f} \equiv\left(H_{f}, \mathbf{P}_{f}\right)
$$

is the operator

$$
P_{f}=k H_{r}{ }^{0}
$$

Here,

$$
k \equiv\left(\left(|\mathbf{k}|^{2}+1\right)^{1 / 2}, \mathbf{k}\right),
$$

and the free "reduced Hamiltonian" $H_{r}{ }^{0}$ acts only on the fiber (i.e., it can be written $H_{r}^{0}=1 \otimes \tilde{\mathscr{H}}_{r}^{0}$ in the tensor product decomposition of $\left.\overline{\mathscr{H}}\right)$ and commutes with $U_{f}(\Lambda)$. Accordingly, one has the relation

$$
U_{f}(\Lambda) k U_{f}(\Lambda)^{*}=\Lambda^{-1} k .
$$

(Here as in the sequel we use the same symbol for an operator $A$ on $\mathscr{H}$ and its transform $I A I^{-1}$ on $\overline{\mathscr{H}}$. Moreover, if $A=1 \otimes \tilde{A}$ on $\overline{\mathscr{H}}$ we will use $A$ to denote both $A$ and $\tilde{A}$. It will be clear from the context which possibility is meant.) It now turns out that a large class of nontrivial operators $V$ exists that act only on the fiber and that just like $H_{r}{ }^{0}$ commute with $U_{f}(\Lambda)$. (Here we have used our assumption that the particles have zero spin: If some of the particles have nonzero spin, one does get a large class of decomposable $V$ that commute with $U_{f}(\Lambda)$, but their fibers $V(\mathbf{k})$ are no longer $\mathbf{k}$ independent. However, it is readily seen that the following considerations apply to this case as well.) Hence, setting

$$
H_{r} \equiv H_{r}^{0}+V
$$

and

$$
P \equiv k H_{r},
$$

it follows from (1.6) that

$$
U_{f}(\Lambda) P U_{f}(\Lambda)^{*}=\Lambda^{-1} P .
$$

Assuming from now on that $V$ is such that the reduced Hamiltonian $H_{r}$ defines a positive operator on $\tilde{\mathscr{H}}$ we can introduce the Abelian $n+1$-parameter unitary group

$$
U(a)=\exp (i a \cdot P)
$$

where

$$
a \cdot P=a_{0} H-\mathbf{a} \cdot \mathbf{P}
$$

We claim that (1.9) implies

$$
U_{f}(\Lambda) U(a) U_{f}(\Lambda)^{*}=U(\Lambda a) .
$$


Indeed, this relation holds for vectors of the form $f(\mathbf{k}) \otimes \psi$ where $\psi$ is an analytic vector for $H_{r}$ and $f$ has compact support, since the power series for $U(\bar{a})$ converges to $U(\bar{a})$ for any $\bar{a}$ on these vectors. Hence, (1.12) follows by linearity and continuity. Thus, if we define

$$
U(a, \Lambda)=U(a) U_{f}(\Lambda)
$$

then the $U(a, \Lambda)$ form a unitary positive energy representation, i.e., one has

$$
U\left(a_{1}, \Lambda_{1}\right) U\left(a_{2}, A_{2}\right)=U\left(a_{1}+\Lambda_{1} a_{2}, \Lambda_{1} \Lambda_{2}\right) .
$$

In the framework first introduced in Ref. [32] and widely discussed since, one writes $H_{f}$ as a square-root operator $\left(\left|\mathbf{P}_{f}\right|^{2}+M_{f}^{2}\right)^{1 / 2}$ and then replaces the invariant mass $M_{f}$ by an operator $M_{\text {int }}$ acting only on internal momenta. The boost generators are then also modified, in such a fashion that the modified generators formally satisfy the commutation relations of the Lie algebra of the Poincaré group. Apart from the nontrivial domain problems that would need to be settled for the resulting expressions to make rigorous sense there is an additional problem of rigor in this setup: the mere fact that a set of unbounded self-adjoint operators represent the Lie algebra of a Lie group (on a dense set) does by no means imply that they give rise to a representation of the group by exponentiation (cf. Ref. [38, Chap. VIII 5]). Although conditions for this to hold are known [39], it is not easy to verify these conditions in concrete cases. In the present scheme this difficulty does not arise: since we do not modify $U_{f}(\Lambda)$ the desired relation (1.14) is an easy consequence of (1.9), as we have shown above.

However, on a more formal level the two approaches lead to similar results: in the center-of-mass rest frame one has $\mathbf{P}_{f}=0$, so that the operator $M_{\text {int }}$ mentioned above corresponds to our operator $H_{r}$. If one chooses $M_{\mathrm{int}}=H_{r}$ as operators on the respective fibers, it is not hard to see from Coester's results [35] that the same $S$-operators will arise in the case of only one scattering channel and no bound states. Indeed, in this case one can set up a unitary equivalence between the two schemes via the respective wave operators. In the general case the situation is less clear.

Lêt us now consider the Lorentz invariance of the $S$-operator, assuming for simplicity that $V$ is such that the reduced wave operators

$$
\tilde{W}_{ \pm}=\lim _{t \rightarrow \pm \infty} \exp \left(i H_{r} t\right) \exp \left(-i H_{r}^{0} t\right)
$$

exist and are unitary. (Thus, there is only one channel and all particles are asymptotically free.) Since

$$
\begin{gathered}
H_{f}=k_{0} H_{r}^{0}, \\
H=k_{0} H_{r},
\end{gathered}
$$

and $k_{\mathbf{0}} \geqslant 1$ for any $\mathbf{k},(1.15)$ and the dominated convergence theorem imply the existence and unitarity of the wave operators

$$
W_{ \pm}-\lim _{t \rightarrow \pm \infty} \exp (i H t) \exp \left(-i H_{f} t\right)
$$


and one has

$$
W_{ \pm}=1 \otimes \tilde{W}_{ \pm}
$$

We claim that

$$
W_{ \pm} U_{f}(a, \Lambda)=U(a, \Lambda) W_{ \pm} .
$$

Taking this for granted, it is clear that the $S$-operator

$$
S=W_{+}^{*} W_{-}
$$

satisfies

$$
S U_{f}(a, \Lambda)=U_{f}(a, \Lambda) S
$$

and consequently is Lorentz invariant.

To prove (1.19) we observe that (1.15) implies

$$
\tilde{W}_{ \pm} H_{r}^{0}=H_{r} \tilde{W}_{ \pm}
$$

But then we have from (1.18) that

$$
W_{ \pm} P_{f}=P W_{ \pm}
$$

from which

$$
W_{ \pm} U_{f}(a)=U(a) W_{ \pm}
$$

Moreover, $H_{r}{ }^{\circ}$ and $H_{r}$ commute with $U(\Lambda)=U_{f}(\Lambda)$, and therefore the operators $\tilde{W}_{ \pm}$do, so that by $(1.18)$

$$
W_{ \pm} U_{f}(\Lambda)=U(\Lambda) W_{ \pm}
$$

concluding the proof.

At the level of classical mechanics we expect that a similar picture obtains. The Poincaré group can be represented by a group of canonical transformations (denoted again by $U_{f}(a, A)$ ) on the phase space $\Omega$ of the $N$ particle positions and momenta $\left(\mathbf{x}_{1}, \ldots, \mathbf{x}_{N} ; \mathbf{p}_{1}, \ldots, \mathbf{p}_{N}\right) \in R^{2 n N}$. The one-parameter subgroups of the representation are the flows generated by Hamiltonian vector fields derived in a well-known fashion (cf. e.g., Refs. [40, 4l]) from $C^{\infty}$ functions on $\Omega$ that represent the Poincaré Lie algebra under Poisson brackets. Explicitly, the functions on $\Omega$ generating time and space translations are given by (1.1), while the functions $M^{\mu v}$, defined by

$$
\begin{array}{rlrl}
M^{k l} & =\sum_{i=1}^{N}\left(x_{i}{ }^{k} p_{i}{ }^{l}-x_{i}{ }^{l} p_{i}{ }^{k}\right), & k \neq l=1, \ldots, n, \\
M^{0 k}=\sum_{i=1}^{N} x_{i}{ }^{k} E_{\mathfrak{D}_{i}}, & k=1, \ldots, n,
\end{array}
$$


represent the customary basis for the Lie algebra of the Lorentz group. The transformation leading to (1.4) is a "point transformation" to new generalized momenta, and may be extended (cf. e.g., Ref. [42]) to a canonical transformation. One then replaces the reduced Hamiltonian $H_{r}^{0}$, which is a function on the reduced phase space $\widetilde{\Omega}$ of internal variables (depending only on the internal momenta), by (1.7) where $V$ is a $C^{\infty}$ function (for instance) on $\tilde{\Omega}$ that commutes with the Lorentz group, i.e.,

$$
V \cdot U_{f}(\Lambda)=V
$$

or equivalently

$$
\left\{V, M^{\mu \nu}\right\}=0 .
$$

The functions $M^{\mu \nu}$ and $P$ (defined in (1.8)) then constitute a new representation of (a basis of) the Lie algebra of the Poincaré group.

However, just as in quantum mechanics one cannot in general conclude from this that there is a corresponding representation of the group. The reason in this case is that the vector fields corresponding to $H$ and $\mathbf{P}$ may fail to be complete, that is, the corresponding local flows may not give rise to global flows. Again, it is much easier to deal with this in the present framework than in the framework introduced in Ref. [32] and used by many authors since. In view of the product form (1.8) and the fact that we do not change the Lorentz group generators, both the $M^{\mu \nu}$-flows and the

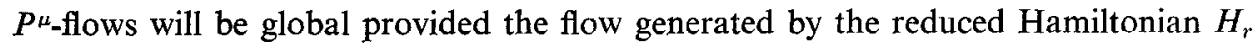
on the reduced phase space $\widetilde{\Omega}$ is global. (To see this, first observe that the flows generated by the $M^{\mu v}$ are global. Indeed, this is obvious for the $M^{k l}$, while the $M^{0 k}$ generate global flows

$$
\begin{aligned}
& \exp \left(\alpha M^{0 k}\right)\left(x_{i}{ }^{k} ; p_{i}^{k}\right) \\
& \quad=\left(x_{i}{ }^{k}\left[\cosh \alpha-\left(p_{i}{ }^{k} / E_{p_{i}}\right) \sinh \alpha\right]^{-1} ; p_{i}^{k} \cosh \alpha-E_{p_{i}} \sinh \alpha\right) .
\end{aligned}
$$

Now note that the factor $k$ in (1.8) only acts as a rescaling of the "time" in Hamilton's equations for the internal variables.) If the potential energy $V$ is bounded below, globality of the reduced flow easily follows from energy conservation. In physical terms, in this case the "reduced particles" cannot escape to infinity in a finite time.

Assuming that $H_{r}$ generates a global flow, we therefore get global flows for $M^{\mu v}$ and $P^{\mu}$. It can be shown that this implies one gets a corresponding representation of the group by canonical transformations. (This is a consequence of the following known fact, which we learned from Mather: If a set of complete $C^{\infty}$ vector fields $X_{i}, i=$ $1, \ldots, L$, on a differentiable manifold $M$ represents a basis $Y_{i}, i=1, \ldots, L$ of the Lie algebra of a simply connected Lie group $G$ under Lie brackets, then there is a unique representation $\pi(G)$ of $G$ by diffeomorphisms on $M$ such that $\pi\left(\exp t Y_{i}\right)$ has generator $X_{i}, i=1, \ldots, L$. Sketch of proof: Regarding the $Y_{i}$ as right invariant vector fields on $G$ one constructs an integrable distribution $\Delta$ on $G \times M$, where $\Delta$ is spanned by $\left(Y_{i}, X_{i}\right)_{(g, m)}, i=1, \ldots, L$. By Frobenius' integrability theorem this gives rise to a 
foliation of $G \times M$ by maximal integral manifolds of $\Delta$. Now the map $\phi(\mathbf{t})=$ $\exp \left(t_{L} Y_{L}\right) \cdots \exp \left(t_{1} Y_{1}\right) e$ has rank $L$ at the origin, and therefore maps a neighborhood of the origin diffeomorphically onto a neighborhood of the identity $e$ in $G$. Thus one can write any $g \in G$ as $g=\phi\left(\mathbf{t}^{n}\right) \cdots \phi\left(\mathbf{t}^{1}\right)$. Since the $X_{i}$ are complete, one can use the flows $\exp \left(t X_{i}\right)$ to construct for any $g \in G$ piecewise continuous curves in $G \times M$ going from $(e, m)$ to $\left(g, m^{\prime}\right)$ for some $m^{\prime}$, whose tangent vectors belong to $\Delta$. Thus, these curves belong to the leaf through $(e, m)$. It readily follows that each leaf of the foliation is a covering space for $G$. But $G$ is simply connected, so that for a fixed $g \in G$ there is one and only one $m^{\prime} \in M$ such that $\left(g, m^{\prime}\right)$ belongs to the leaf through $(e, m)$. Now define $\pi(g) m=m^{\prime}$. Then $\pi\left(g_{1} g_{2}\right)=\pi\left(g_{1}\right) \pi\left(g_{2}\right)$ since the map $(g, m) \rightarrow$ $\left(g g_{2}^{-1}, m\right)$ permutes the leaves. It is easy to verify the other properties.)

The classical scattering properties are also analogous to the quantum case. Let us assume for example that $V$ is a rapidly decreasing function of the internal generalized coordinates and that the reduced wave operators

$$
\tilde{W}_{ \pm}=\lim _{t \rightarrow \pm \infty} \exp \left(-t H_{r}\right) \exp \left(t H_{r}{ }^{0}\right)
$$

exist on appropriate subsets of $\tilde{\Omega}$ (for the wave operator formalism in classical mechanics see, e.g., Refs. $[22,40])$. Then the wave operators

$$
W_{ \pm}=\lim _{t \rightarrow \pm \infty} \exp (-t H) \exp \left(t H_{f}\right)
$$

also exist on the corresponding subsets of $\Omega$, and (1.18) holds true. This is not as immediate as in the quantum case, but can be seen as follows. Denoting by $\mathbf{x}$ the generalized coordinate conjugate to $\mathbf{k}$ and by $(\tilde{y}, \tilde{q})$ a point of $\widetilde{\Omega}$, the Hamilton equations and the fact that $\mathbf{P}$ and $\mathbf{P}_{f}$ commute with $H$ resp. $H_{f}$ imply that

$$
\exp (t H)(\mathbf{x}, \mathbf{k} ; \tilde{y}, \tilde{q})=\left(\mathbf{x}+t \mathbf{k} k_{0}^{-1}\left(H_{r}^{0}(\tilde{q})+V(\tilde{y})\right), \mathbf{k} ; \exp \left(k_{0} t H_{r}\right)(\tilde{y}, \tilde{q})\right)
$$

resp.

$$
\exp \left(t H_{f}\right)(\mathbf{x}, \mathbf{k} ; \tilde{y}, \tilde{q})=\left(\mathbf{x}+t \mathbf{k} k_{0}^{-1} H_{r}^{0}(\tilde{q}), \mathbf{k} ; \exp \left(k_{0} t H_{r}^{0}\right)(\tilde{y}, \tilde{q})\right)
$$

Noting that

$$
\exp \left(t H_{r}^{0}\right)(\tilde{y}, \tilde{q})=(\tilde{y}+t v(\tilde{q}), \tilde{q})
$$

(where $v$ is the $\tilde{q}$-gradient of $H_{r}^{0}(\tilde{q})$ ) we conclude

$$
\begin{aligned}
\exp (-t H) \exp \left(t H_{f}\right)(\mathbf{x}, \mathbf{k} ; \tilde{y}, \tilde{q}) \\
\quad=\left(\mathbf{x}-t \mathbf{k} k_{0}^{-1} V\left(\tilde{y}+t k_{0} v(\tilde{q})\right), \mathbf{k} ; \exp \left(-k_{0} t H_{r}\right) \exp \left(k_{0} t H_{r}^{0}\right)(\tilde{y}, \tilde{q})\right) \\
\quad \rightarrow\left(\mathbf{x}, \mathbf{k} ; \tilde{W}_{ \pm}(\tilde{y}, \tilde{q})\right)
\end{aligned}
$$


for $t \rightarrow \pm \infty$, provided $\tilde{W}_{ \pm}$are defined on $(\tilde{y}, \tilde{q})$. Using (1.18) the relations (1.19) now follow as in the quantum case from the intertwining relations for $\tilde{W}_{ \pm}$and the fact that $\tilde{W}_{ \pm}$commute with $U_{f}(\Lambda)$. Thus the $S$-operator

$$
S=W_{+}^{-1} W_{-}
$$

is Lorentz invariant, i.e., satisfies (1.21).

\subsection{Outline of the Paper}

In the classical case we only work out the explicit details of the above considerations for a simple illustrative case, viz., the siluation of two equal mass particles on the line that interact via a class of attractive potentials. (As in the quantum case, in one dimension it is actually more convenient to give a formulation in terms of the particle rapidities.) We show that in this case the $S$-operator is completely characterized by an invariant phase shift that, conversely, uniquely determines the potential in the class defined below. These results can be found in Section 2.2, while Section 2.1 treats the quantum case. Here too, the detailed discussion is restricted to a simple class of interactions, which exemplifies the results that might be obtained for more general interactions.

In the rest of the paper (Sections 3-5) only the quantum case is studied. As in Section 2 we begin each section by describing the transformation that brings the free momentum operator in the product form (1.4), the action of the Lorentz group in the new variables, and interactions that are allowed.

In Section 3 we consider $N$ equal mass particles on the line. In preparation for a more detailed study of the three-dimensional $N$-body problem in Section 5 we consider some difficulties connected with "pair potentials," which are already apparent in this simpler case. Furthermore, it is pointed out that in one dimension both nonrelativistic $S$-operators and $S$-operators of relativistic quantum field theories lack the usual cluster properties (cf. Refs. [22, 43]), so that failure of clustering in the present scheme is not surprising.

Section 4 deals with the case of two spin-zero particles in three space dimensions. As in Section 2.1 a fully satisfactory theory is shown to result if the interaction is suitably restricted.

In Section 5 we study the general case of $N$ particles with arbitrary masses and spins in three space dimensions. We point out a natural analog of the pair potentials of Schrödinger theory and study the scattering theory for this interaction. The nonlinearity of the coordinate change to center-of-mass and internal variables and the replacement of the nonrelativistic kinetic energy $|\mathbf{q}|^{2}$ by the nonquadratic relativistic energy $\left(|\mathbf{q}|^{2}+m^{2}\right)^{1 / 2}$ lead to mathematical and physical difficulties not present at the nonrelativistic level. In particular, it seems unlikely that one can introduce pair potentials for which the $S$-operator has the cluster properties known to hold in threedimensional nonrelativistic quantum mechanics $[22,44]$ and axiomatic quantum field theory [45]. (The fact that the introduction of interaction in a scheme of the type 
considered here leads to unphysical properties has been recognized before in the Bakamjian-Thomas-Foldy framework, and has been particularly emphasized by Foldy [33].)

Already at this point it will be clear to the reader that the present scheme does not naturally lead to antiparticles, since it lacks by construction the negative energy states of relativistic wave equations that lead to antiparticles upon second quantization. Also, again by construction, the dynamics does not give rise to particle production (although of course breakup collisions might occur in the case of more than one channel). Nevertheless, it is possible to define positive Hamiltonians providing a fully relativistic description of atomic systems that is presumably quite accurate in the energy regime in which particle production may be neglected. In particular, we argue that their bound-state levels are in closer agreement with observation than the corresponding nonrelativistic levels. The choice of these Hamiltonians and the arguments for their accuracy are mainly based on the Dirac equation, which is well-known to give a better description of experimental reality than the nonrelativistic Coulomb interaction. The last half of Section 5 is devoted to a detailed discussion of these operators and their higher-spin generalizations. $\Lambda$ number of rigorous results is obtained, but energy levels are also studied in formal perturbation theory. Finally, we briefly consider the question of how one could couple a quantized radiation field in the spin$1 / 2$ case.

In Sections 4 and 5 we have occasion to use some known results concerning the Lorentz and Poincaré groups that we have summarized in an Appendix.

We would like to add a comment on the presentation of the material. Although our discussion is mathematically rigorous (unless indicated otherwise) we have chosen to adopt a rather informal style in comparison to the "theorem/proof" style of modern mathematical physics. We hope that this will make the paper more accessible to the theoretical physicist to whom this paper may be of some interest, since it raises questions in theoretical physics in addition to leading to interesting problems in mathematical physics (cf. especially Section 5). However, we have not attempted to detail the modern tools of mathematical physics that have been used in some parts of the paper, and instead refer the reader to the books by Reed and Simon [22, 37, 38, 46], where these methods have been so well summarized. We have also been rather sketchy in the proofs of some of our assertions (in particular in Section 5), since a more complete account would have considerably increased the size of this paper, and might have obscured the main line of reasoning.

\section{Two Equal Mass Particles in One Dimension}

In this section we consider two equal mass relativistic particles on the line. For convenience we assume their masses are 1/2. In Section 2.1 we consider the particles in a quantum-mechanical setting, in Section 2.2 in the framework of classical mechanics. 


\subsection{Quantum Mechanics}

We shall take as the state space of the particles the Hilbert space $\mathscr{H} \equiv L^{2}\left(d \theta_{1} d \theta_{2}\right)$, i.e., the space of square-integrable functions depending on the particle rapidities $\theta_{1}$ and $\theta_{2}$. The use of rapidities instead of momenta is very convenient in the equal-mass case, but of course these two choices are equivalent: The space $\mathscr{H}^{\prime} \equiv L^{2}\left(d p_{1} d p_{2}\right)$ of square-integrable functions depending on the particle momenta $p_{1}$ and $p_{2}$ is mapped onto $\mathscr{H}$ by the unitary operator

$$
(U f)\left(\theta_{1}, \theta_{2}\right)=\frac{1}{2}\left[\cosh \theta_{1} \cosh \theta_{2}\right]^{1 / 2} f\left(\frac{1}{2} \sinh \theta_{1}, \frac{1}{2} \sinh \theta_{2}\right)
$$

with inverse

$$
\begin{aligned}
& \left(U^{-1} g\right)\left(p_{1}, p_{2}\right) \\
& \quad=2\left[\left(1+4 p_{1}{ }^{2}\right)\left(1+4{p_{2}}^{2}\right)\right]^{-1 / 4} g\left(\ln \left[2 p_{1}+\left(1+4 p_{1}{ }^{2}\right)^{1 / 2}\right], \ln \left[2 p_{2}+\left(1+4 p_{2}{ }^{2}\right)^{1 / 2}\right]\right) .
\end{aligned}
$$

These formulas can be used by readers wishing to translate the results into "momenta language."

In terms of rapidities the free Hamiltonian, momentum operator and boost generator are given by

$$
\begin{aligned}
H_{f} & =\frac{1}{2} \cosh \theta_{1}+\frac{1}{2} \cosh \theta_{2}, \\
\mathbf{P}_{f} & =\frac{1}{2} \sinh \theta_{1}+\frac{1}{2} \sinh \theta_{2}, \\
L_{f} & =i \partial_{\theta_{1}}+i \partial_{\theta_{2}} .
\end{aligned}
$$

They generate the free representation of the Poincaré group in one dimension, denoted by $U_{f}(a, \Lambda)$ :

$$
\begin{aligned}
\left(U_{f}(a, \Lambda) f\right)\left(\theta_{1}, \theta_{2}\right)= & \exp \left[i a_{0}\left(\frac{1}{2} \cosh \theta_{1}+\frac{1}{2} \cosh \theta_{2}\right)\right. \\
& \left.-i a_{1}\left(\frac{1}{2} \sinh \theta_{1}+\frac{1}{2} \sinh \theta_{2}\right)\right] \cdot f\left(\theta_{1}-\alpha, \theta_{2}-\alpha\right) .
\end{aligned}
$$

Here, $\alpha$ is the rapidity change corresponding to $\Lambda$, i.e.,

$$
A=\left(\begin{array}{cc}
\cosh \alpha & \sinh \alpha \\
\sinh \alpha & \cosh \alpha
\end{array}\right)
$$

Proceeding as sketched in Section 1.1, we now transform to center-of-mass variables

$$
\begin{gathered}
\phi=\left(\theta_{1}+\theta_{2}\right) / 2, \\
\theta=\left(\theta_{1}-\theta_{2}\right) / 2
\end{gathered}
$$

and we map $\mathscr{H}$ onto $\overline{\mathscr{H}} \equiv L^{2}(d \phi d \theta)$ by means of the unitary operator $I$ satisfying

$$
\begin{aligned}
(I f)(\phi, \theta) & \equiv 2^{1 / 2} f(\phi+\theta, \phi-\theta), \\
\left(I^{-1} g\right)\left(\theta_{1}, \theta_{2}\right) & =2^{-1 / 2} g\left(\left(\theta_{1}+\theta_{2}\right) / 2,\left(\theta_{1}-\theta_{2}\right) / 2\right) .
\end{aligned}
$$


On $\overline{\mathscr{H}}$ we then get

$$
\begin{aligned}
& H_{f}=\cosh \phi \cosh \theta, \\
& \mathbf{P}_{f}=\sinh \phi \cosh \theta, \\
& L_{f}=i \partial_{\phi},
\end{aligned}
$$

$\left(U_{f}(a, \Lambda) g\right)(\phi, \theta)=\exp \left[i a_{0} \cosh \phi \cosh \theta-i a_{1} \sinh \phi \cosh \theta\right] f(\phi-\alpha, \theta)$.

Clearly, the operators in (2.7) are in the fibered form alluded to in Section 1.1. Here, $U_{f}(\Lambda)$ acts as the identity on the fiber, i.e., $U_{f}(\Lambda) \simeq \bar{U}_{f}(\Lambda) \otimes 1$ in the tensor-product decomposition $\overline{\mathscr{H}} \simeq L^{2}(d \phi) \otimes L^{2}(d \theta)$. Hence, setting

$$
\begin{gathered}
H=\cosh \phi(\cosh \theta+\lambda V), \quad \lambda \in R \\
\mathbf{P}=\sinh \phi(\cosh \theta+\lambda V), \\
U(a, \Lambda)=\exp (\text { ia } \cdot P) U_{f}(\Lambda),
\end{gathered}
$$

the $U(a, A)$ will represent the Poincare group provided $V$ acts on the fiber only. Assuming this from now on we have (symbolically in general)

$$
(V f)(\phi, \theta)-\int d \theta^{\prime} K\left(\theta, \theta^{\prime}\right) f\left(\phi, \theta^{\prime}\right)
$$

Of course, we also require $V$ to be such that the reduced Hamiltonian

$$
H_{r} \equiv \cosh \theta+\lambda V
$$

defines a self-adjoint operator on the fiber $L^{2}(d \theta)$. If the kernel $K$ of $V$ is sufficiently regular, the analysis of the operators $H$ and $H_{r}$ presents little difficulty, and proceeds along well-known lines [37]. For example, if

$$
K\left(\theta, \theta^{\prime}\right)=(2 \pi)^{-1} \int d x \exp \left[-i\left(\theta-\theta^{\prime}\right) x\right] F(x)
$$

where $F$ is a real-valued test function in $S(R)$, then $V$ is relatively compact with respect to $\cosh \theta$ so that the essential spectrum of $H_{r}$ is $[1, \infty$ ). (This spectrum is actually purely absolutely continuous for any $\lambda$, which is a consequence of work by Enss [64], discussed in Ref. [22] and extended by Simon in Ref. [65]. We thank B. Simon for pointing this out to us.) Assuming $|F| \leqslant 1$ without loss of generality, the only additional spectrum that $H_{r}$ may have consists of a number of finitely degenerate eigenvalues in the interval $(1-|\lambda|, 1)$, which can be shown to be finite by a comparison argument. The operator $H$ has purely absolutely continuous spectrum in $[1, \infty)$ if $H_{r}$ has no eigenvalues $<1$, in $\left[E_{0}, \infty\right)$ if the smallest eigenvalue $E_{0}$ of $H_{r}$ satisfies $0<E_{0}<1$. If $H_{r}$ has a zero energy bound state, $H$ clearly has an infinitely degenerate eigenvalue zero. If $E_{0}$ is strictly negative, $H$ has purely absolutely continuous spectrum in $\left(-\infty, E_{-}\right] \cup\left[\min \left(1, E_{+}\right), \infty\right)$, where $E_{-}$is the greatest strictly negative eigenvalue of $H_{r}$, and $E_{+}$the smallest strictly positive eigenvalue. The last two possibilities are 
considered unphysical, so one may wish to assume that $\lambda$ and/or $V$ are such that only strictly positive energy bound states occur. Note that $U(a, A)$ restricts to an irreducible representation of the Poincaré group of mass $m$ on $L^{2}(d \phi) \otimes f$ if $f(\theta)$ is an eigenfunction of $H$, with eigenvalue $m>0$.

Under our assumptions on $V$ it also easily follows that the hypothesis of the Kuroda-Birman theorem [22] is satisfied, so that the reduced wave operators exist and are complete. Hence the $S$-operator exists and is unitary. As detailed in Section 1.1 the wave operators intertwine the free and perturbed representations, and therefore $S$ is Lorentz invariant. The action of $S$ is given by

$$
(S f)(\phi, \theta)=t(\theta) f(\phi, \theta)+r(\theta) f(\phi,-\theta),
$$

where the transmission and reflection coefficients are measurable functions satisfying

$$
\begin{aligned}
|t(\theta)|^{2}+|r(-\theta)|^{2} & =1, \\
\bar{t}(\theta) r(\theta)+t(-\theta) \bar{r}(-\theta) & =0 .
\end{aligned}
$$

The conditions on $V$ may be considerably relaxed to still imply essentially the same picture as sketched above, but we leave this to the interested reader.

\subsection{Classical Mechanics}

As the phase space of our two particles we take the four-dimensional Euclidean space

$$
\Omega=\left\{\left(r_{1}, r_{2} ; \theta_{1}, \theta_{2}\right) \in R^{4}\right\}
$$

with its natural 2-form $\omega=d r_{1} \wedge d \theta_{1}+d r_{2} \wedge d \theta_{2}$. Again the $\theta_{i}$ are the particle rapidities. The canonical transformation

$$
\begin{aligned}
& x_{i}=2 r_{i} / \cosh \theta_{i}, \\
& p_{i}=\frac{1}{2} \sinh \theta_{i},
\end{aligned} \quad i=1,2
$$

leads to a description in terms of particle momenta $p_{i}$ and shows the relation between the generalized coordinates $r_{i}, \theta_{i}$ and the "physical" position coordinates $x_{i}$.

The functions

$$
\begin{aligned}
& H_{f}=\frac{1}{2} \cosh \theta_{1}+\frac{1}{2} \cosh \theta_{2}, \\
& \mathbf{P}_{f}=\frac{1}{2} \sinh \theta_{1}-\frac{1}{2} \sinh \theta_{2}, \\
& L_{f}=-r_{1}-r_{2}
\end{aligned}
$$

give rise to Hamiltonian vector fields that generate global flows of canonical transformations. Combining these in an obvious way one obtains the free representation of the Poincaré group by canonical transformations on $\Omega$ :

$$
U_{f}(a, A)\left(r_{i} ; \theta_{i}\right)=\left(r_{i}+\frac{1}{2} a_{0} \sinh \left(\theta_{i}+\alpha\right)-\frac{1}{2} a_{1} \cosh \left(\theta_{i}+\alpha\right) ; \theta_{i}+\alpha\right),
$$

where $i=1,2$ and $\alpha$ is the rapidity corresponding to $\Lambda$. 
Making a canonical transformation to center-of-mass variables

$$
\begin{array}{ll}
s=r_{1}+r_{2}, & \phi=\left(\theta_{1}+\theta_{2}\right) / 2, \\
d=r_{1}-r_{2}, & \theta=\left(\theta_{1}-\theta_{2}\right) / 2,
\end{array}
$$

one gets

$$
\begin{gathered}
H_{f}=\cosh \phi \cosh \theta, \\
\mathbf{P}_{f}=\sinh \phi \cosh \theta, \\
L_{f}=-s, \\
U_{f}(a, \Lambda)(s, d ; \phi, \theta)=\left(s+\left[a_{0} \sinh (\phi+\alpha)-a_{1} \cosh (\phi+\alpha)\right] \cosh \theta,\right. \\
\left.d+\left[a_{0} \cosh (\phi+\alpha)-a_{1} \sinh (\phi+\alpha)\right] \sinh \theta ; \phi+\alpha, \theta\right) .
\end{gathered}
$$

If we now set

$$
\begin{aligned}
& H=\cosh \phi(\cosh \theta+V(d, \theta)), \\
& \mathbf{P}=\sinh \phi(\cosh \theta+V(d, \theta)), \\
& L=-s,
\end{aligned}
$$

where $V$ is real valued and $C^{\infty}$, then it is clear that these functions again represent the Lie algebra of the Poincaré group.

As in Section 2.1 we shall study the dynamics and scattering only for a simple, yet large, class of potentials $V$ to which standard techniques [22, 47] can be casily applied. We propose to consider functions $V$ that are rapidly decreasing test functions of $d$ only and that are even, nonpositive, and monotone on $[0, \infty)($ e.g., $V=-\cosh d)$. We denote this class by $\mathscr{P}$. For any $V \in \mathscr{P}$ an application of the contraction mapping principle combined with energy conservation gives global existence and uniqueness of solutions to the Hamilton equations

$$
\begin{aligned}
& \dot{d}=\sinh \theta, \\
& \dot{\theta}=-V^{\prime}(d)
\end{aligned}
$$

for the reduced Hamiltonian

$$
H_{r}(d, \theta)=\cosh \theta+V(d)
$$

on the reduced phase space

$$
\widetilde{\Omega}=\left\{(d ; \theta) \in R^{2}\right\} .
$$

Correspondingly, one gets a global flow on $\widetilde{\Omega}$.

$$
\exp \left(t H_{r}\right)(d ; \theta) \equiv(D(t, d, \theta) ; \Theta(t, d, \theta)),
$$


and three global flows on $\Omega$,

$$
\begin{aligned}
\exp (t H)(s, d ; \phi, \theta)= & \left(s+t \sinh \phi H_{r}(d, \theta), D(t \cosh \phi, d, \theta) ;\right. \\
& \phi, \Theta(t \cosh \phi, d, \theta)), \\
\exp (t \mathbf{P})(s, d ; \phi, \theta)= & \left(s+t \cosh \phi H_{r}(d, \phi), D(t \sinh \phi, d, \theta) ;\right. \\
& \phi, \Theta(t \sinh \phi, d, \theta)), \\
\exp (t L)(s, d ; \phi, \theta)= & (s, d ; q+t, \theta) .
\end{aligned}
$$

Hence, setting

$$
U(a, \Lambda)=\exp \left(a_{0} H-\mathbf{a} \cdot \mathbf{P}\right) \exp (\alpha L)
$$

the $U(a, A)$ represent the Poincaré group (cf. Section 1.1).

For $V \in \mathscr{P}$ arguments similar to those in Refs. [22, 40,47] imply that the reduced wave operators (1.30) exist and are canonical transformations from

$$
\widetilde{\Omega_{0}}=\tilde{\Omega} \backslash\{\theta=0\}
$$

onto the subset of $\widetilde{\Omega}$ where the "reduced particle" is unbound, i.e., the set

$$
\widetilde{\Omega}_{\mathrm{in}}=\widetilde{\Omega}_{\mathrm{out}}=\widetilde{\Omega} \backslash\left\{(d ; \theta) \mid H_{r}(d, \theta) \leqslant 1\right\} .
$$

Therefore, the reduced $S$-operator

$$
\tilde{S}=\tilde{W}_{+}^{-1} \tilde{W}_{-}
$$

is a canonical transformation from $\widetilde{\Omega}_{0}$ onto $\widetilde{\Omega}_{0}$. We set

$$
\tilde{S}(d ; \theta)=(d \mid \delta(d, \theta) ; \bar{\theta}(d, \theta)) \text {. }
$$

Now from the intertwining relations for $\tilde{W}_{ \pm}$it follows that $\tilde{S} \exp (t \cosh \theta)=$ $\exp (t \cosh \theta) \tilde{S}$. Since $\widetilde{S}$ is canonical, this implies $\cosh \theta \cdot \tilde{S}=\cosh \theta$, so that $\cosh \tilde{\theta}(d, \theta)=\cosh \theta$. Because our potentials do not reflect unbound particles we conclude that $\bar{\theta}(d, \theta)=\theta$. But then $\theta \cdot \tilde{S}=\theta$, from which $\exp (t \theta) \tilde{S}=\tilde{S} \exp (t \theta)$, since $\tilde{S}$ is canonical. It follows from this that $d+\delta(d, \theta)+t=d+t+\delta(d+t, \theta)$, so that $\delta$ is a function of $\theta$ only. As a result of this argument we obtain

$$
\tilde{S}(d ; \theta)=(d+\delta(\theta) ; \theta) \text {. }
$$

The evenness of $V$ is easily seen to imply that $\delta$ is odd.

Summarizing, we conclude (cf. Section 1.1) that the wave operators (1.31) have domain

$$
\Omega_{0}=\Omega \backslash\{\theta=0\}
$$

and range

$$
\Omega_{\mathrm{in}}=\Omega_{\mathrm{out}}=\{(s, d ; \phi, \theta) \in \Omega \mid \cosh \theta+V(d)>1\}
$$


and satisfy (1.19). The $S$-operator (1.36) satisfies (1.21) and is given by

$$
S(s, d ; \phi, \theta)=(s, d+\delta(\theta) ; \phi, \theta)
$$

on its domain $\Omega_{0}$. Here, $\delta$ is an odd function of $\theta$ on $R \backslash\{0\}$. In the original variables one has

$$
S\left(r_{1}, r_{2} ; \theta_{1}, \theta_{2}\right)=\left(r_{1}+\frac{1}{2} \delta\left(\frac{1}{2}\left(\theta_{1}-\theta_{2}\right)\right), r_{2}+\frac{1}{2} \delta\left(\frac{1}{2}\left(\theta_{2}-\theta_{1}\right)\right) ; \theta_{1}, \theta_{2}\right),
$$

while in terms of the positions and momenta (2.15) one gets

$S\left(x_{1}, x_{2} ; p_{1}, p_{2}\right)=\left(x_{1}+\delta\left(\frac{1}{2}\left(\theta_{1}-\theta_{2}\right)\right) / \cosh \theta_{1}, x_{2}+\delta\left(\frac{1}{2}\left(\theta_{2}-\theta_{1}\right)\right) / \cosh \theta_{2} ; p_{1}, p_{2}\right)$,

where

$$
\theta_{i}\left(p_{i}\right) \equiv \ln \left[2 p_{i}+\left(1+4 p_{i}^{2}\right)^{1 / 2}\right]
$$

Note that in (2.35) the invariant phase shift is multiplied by Lorentz-Fitzgerald contraction factors.

We shall finish this section by deriving an expression for $\delta(\theta)$ in terms of $V(d)$, and by showing that there is a $1-1$ correspondence between potentials $V \in \mathscr{P}$ and their phase shifts $\delta_{V}$.

Choose $(d, \theta)$ with $\theta>0$ and set

$$
\tilde{W}_{-}(d, \theta) \equiv(\bar{d}, \bar{\theta}) \text {. }
$$

Then one has from (2.24) and (2.33)

$$
\lim _{t \rightarrow-\infty}(D(t, \bar{d}, \bar{\theta})-d-t \sinh \theta)=0
$$

resp.

$$
\lim _{t \rightarrow \infty}(D(t, \bar{d}, \bar{\theta})-d-\delta(\theta)-t \sinh \theta)=0 .
$$

In view of the relation

$$
\begin{gathered}
{[D(t, \bar{d}, \bar{\theta})-t \sinh \theta]-[D(-t, \bar{d}, \bar{\theta})-(-t) \sinh \theta]} \\
=\int_{-t}^{t}[\dot{D}(\tau, \bar{d}, \bar{\theta})-\sinh \theta] d \tau
\end{gathered}
$$

we then conclude that

$$
\delta(\theta)=\int_{-\infty}^{\infty}[\dot{D}(t, \vec{d}, \bar{\theta})-\sinh \theta] d t
$$

Since $\Theta(t, \vec{d}, \bar{\theta})>0$ for all $t$ (by energy conservation), we have from (2.21) that $\bar{D}(t, \bar{d}, \bar{\theta})>0$ for all $t$. Hence we may regard $t$ as a function of $D, \bar{d}, \bar{\theta}$ :

$$
t=T(D, d, \bar{\theta})
$$


and change variables in (2.41):

$$
\delta(\theta)=\int_{-\infty}^{\infty}[1-\sinh \theta / \sinh \Theta(T(D, \bar{d}, \bar{\theta}), \bar{d}, \bar{\theta})] d D .
$$

Now the intertwining relations for $\tilde{W}_{-}$imply that

$$
\cosh \theta=\cosh \bar{\theta}+V(\bar{d})
$$

from which, by energy conservation,

$$
\cosh \theta=\cosh \Theta(T(D, \bar{d}, \bar{\theta}), \bar{d}, \bar{\theta})+V(D), \quad \forall D \in R .
$$

Solving for $\sinh \Theta$ and inserting in (2.34) gives the promised formula:

$\delta(\theta)=\int_{-\infty}^{\infty}\left(1-\left[1-2 \operatorname{coth} \theta V(x) / \sinh \theta+V^{2}(x) / \sinh ^{2} \theta\right]^{-1 / 2}\right) d x, \quad \theta>0$.

We finally prove the $1-1$ correspondence mentioned above. Fix $V \in \mathscr{P}$ and set $\delta \equiv \delta_{V}$. Assume that $\delta_{\bar{p}}=\delta$ for $\bar{V} \in \mathscr{P}$. We have to show that this implies $\bar{V}=V$. First we observe that $\delta(\theta)$ is an analytic function in a neighborhood of the positive real axis since $V$ rapidly vanishes at infinity. Hence, it is uniquely determined by its large $\theta$ behavior. Let us therefore introduce

$$
\sinh \alpha=1 / \sinh \theta \text {. }
$$

In terms of $\alpha$ we have

$$
\tilde{\delta}(\alpha)-\int_{-\infty}^{\infty}\left(1-\left[1-2 \cosh \alpha V(x) \sinh \alpha+(V(x) \sinh \alpha)^{2}\right]^{-1 / 2}\right) d x
$$

from which it easily follows that $\tilde{\delta}(\alpha)$ is analytic in a neighborhood of the origin. The square-root factor is just the generating function for the Legendre polynomials (cf. e.g., Ref. [48]):

$\left[1-2 \cosh \alpha(V(x) \sinh \alpha)+(V(x) \sinh \alpha)^{2}\right]^{-1 / 2}=\sum_{l=0}^{\infty}(V(x) \sinh \alpha)^{l} P_{l}(\cosh \alpha)$.

(This observation is due to Wightman.) Thus, for $|\alpha|$ small enough we have

$$
\tilde{\delta}(\alpha)=-\sum_{l=1}^{\infty} c_{l} \sinh ^{l} \alpha P_{l}(\cosh \alpha),
$$

where

$$
c_{n} \equiv \int_{-\infty}^{\infty} V(t)^{n} d t, \quad n=1,2, \ldots
$$


From our assumption $\delta_{\bar{V}}=\delta$ it readily follows that one also has

$$
c_{n}=\int_{-\infty}^{\infty} \bar{V}(t)^{n} d t, \quad n=1,2, \ldots
$$

We now note that since $V$ is nonpositive,

$$
\int_{-\infty}^{\infty} V(t)^{n} d t=\int_{-\infty}^{0} x^{n} d m_{V}(x),
$$

where $m_{V}(x)$ is the distribution function of $V$ on $(-\infty, 0)$ (i.e., the function

$$
m_{V}(x)=\mu\{t \mid V(t) \leqslant x\},
$$

where $\mu$ is Lebesgue measure), and where the second integral is a Riemann-Stieltjes integral. Setting

$$
a_{n} \equiv-c_{n+1} /(n+1), \quad n=0,1,2, \ldots
$$

it is easy to see that

$$
a_{n}=\int_{-\infty}^{\infty} x^{n} d \rho(x), \quad n=0,1,2, \ldots,
$$

where $\rho$ is the positive measure

$$
d \rho(x)=\chi_{(-\infty, 0)}(x) m_{V}(x) d x .
$$

However, it is clear that the $\left\{a_{n}\right\}_{n=0}^{\infty}$ satisfy the condition for the Hamburger moment problem to have a unique solution [46], so that $\rho$ and hence $m_{\nu}(x)$ are uniquely determined by the "moments" $\left\{c_{n}\right\}_{n=1}^{\infty}$. In view of (2.52) this implies

$$
m_{\bar{V}}(x)=m_{V}(x), \quad \forall x \in(-\infty, 0) .
$$

We finally observe that, since our potentials are by assumption even and monotone on $[0, \infty), m_{V}(x)$ is just twice the inverse function of $V$ on the intervals in $[0, \infty)$ where $V$ is strictly monotone, while its jumps are twice the length of the intervals of constancy. Thus (2.58) implies that $\bar{V}=V$, as claimed.

We have thus shown that there is a $1-1$ correspondence between potentials in $\mathscr{P}$ and their "S$S$-matrices." Note that our discussion shows that the phase shift only detcrmines the distribution function of $V \in \mathscr{P}$, so that any potential with the same "moments" $c_{n}$ as $V$ (and to which the derivations apply) gives rise to the same phase shift as $V$. In particular translates of $V$ have this property. Consequently, there is no uniqueness in the inverse scattering problem. 


\section{3. $N$ Equal-Mass Particles in One Dimension}

We shall now consider $N$ equal mass particles on the line. In this case it is convenient to assume their mass is one. The particles will be treated quantum mechanically.

We take the Hilbert space $\mathscr{H} \equiv L^{2}\left(d \theta_{1} \cdots d \theta_{N}\right)$ as the state space of the particles, where as in Section 2 the $\theta_{i}$ are the particle rapidities. Again, a formulation in terms of their momenta is possible, but this is left to the reader desiring this. The free representation of the Poincaré group on $\mathscr{H}$ is given by

$$
\begin{aligned}
& \left(U_{f}(a, \Lambda) f\right)\left(\theta_{1}, \ldots, \theta_{N}\right) \\
& \quad=\exp \left[i a_{0} \sum_{i=1}^{N} \cosh \theta_{i}-i a_{1} \sum_{i=1}^{N} \sinh \theta_{i}\right] f\left(\theta_{1}-\alpha, \ldots, \theta_{N}-\alpha\right),
\end{aligned}
$$

corresponding to generators

$$
\begin{aligned}
& H_{f}=\sum_{i=1}^{N} \cosh \theta_{i}, \\
& \mathbf{P}_{f}=\sum_{i=1}^{N} \sinh \theta_{i}, \\
& L_{f}=\sum_{i=1}^{N} i \partial_{\theta_{i}} .
\end{aligned}
$$

To get $P_{f}=\left(H_{f}, \mathbf{P}_{f}\right)$ in the desired product form, we write

$$
\begin{aligned}
& \sum_{i} \cosh \theta_{i}=M \cosh \phi, \\
& \sum_{i} \sinh \theta_{i}=M \sinh \phi
\end{aligned}
$$

which implies $M$ must be the invariant mass:

$$
M=\left[\sum_{i, j=1}^{N} \cosh \left(\theta_{i}-\theta_{j}\right)\right]^{1 / 2} .
$$

Solving for $\phi$ we get

$$
\phi(\theta)=\ln \left[\sum_{i=1}^{N} \exp \theta_{i}\right]-\frac{1}{2} \ln \left[\sum_{i, j=1}^{N} \cosh \left(\theta_{i}-\theta_{j}\right)\right] .
$$


Note that for $N=2$ one gets $\phi=\left(\theta_{1}+\theta_{2}\right) / 2$ as in Section 2, and that

$$
\sum_{i=1}^{N} \sinh \left(\theta_{i}-\phi(\theta)\right)=0
$$

i.e., the Lorentz boost to the center-of-mass frame has rapidity $-\phi(\theta)$.

We are now faced with a difficulty that is analogous to a well-known problem in nonrelativistic $N$-body quantum mechanics: there is a natural choice for the center-ofmass variable, but the choice of the variables describing the internal motion is largely arbitrary (except for $N=2$ ). In our case it seems most convenient to choose $N-1$ of the $N$ center-of-mass rapidities, i.e., the rapidities

$$
\bar{\theta}_{i}=\theta_{i}-\phi(\theta), \quad i=1, \ldots, N
$$

(cf. (3.6)). Selecting, e.g., the first $N-1 \bar{\theta}_{i}$ as independent variables, one gets for the corresponding unitary operator $I$ that maps $\mathscr{H}$ onto $\mathscr{H} \equiv L^{2}\left(d \phi d \bar{\theta}_{1} \cdots d \bar{\theta}_{N-1}\right)$

$$
(\text { If })\left(\phi, \bar{\theta}_{1}, \ldots, \bar{\theta}_{N-1}\right)=\left[\left(\sum_{i=1}^{N} \cosh \bar{\theta}_{i}\right) / \cosh \bar{\theta}_{N}\right]^{1 / 2} f\left(\phi+\bar{\theta}_{1}, \ldots, \phi+\bar{\theta}_{N}\right)
$$

and for its inverse

$$
\begin{aligned}
\left(I^{-1} g\right)\left(\theta_{1}, \ldots, \theta_{N}\right)= & {\left[\left(\sum_{i=1}^{N} \cosh \left(\theta_{i}-\phi(\theta)\right)\right) / \cosh \left(\theta_{N}-\phi(\theta)\right)\right]^{-1 / 2} } \\
& \times g\left(\phi(\theta), \theta_{1}-\phi(\theta), \ldots, \theta_{N-1}-\phi(\theta)\right) .
\end{aligned}
$$

In (3.8) $\bar{\theta}_{N}$ is the function

$$
\bar{\theta}_{N}\left(\bar{\theta}_{1}, \ldots, \bar{\theta}_{N-1}\right) \equiv \ln \left[-\sum_{i=1}^{N-1} \sinh \bar{\theta}_{i}+\left(\left(\sum_{i=1}^{N-1} \sinh \bar{\theta}_{i}\right)^{2}+1\right)^{1 / 2}\right] .
$$

Transforming the free representation (3.1) to $\overline{\mathscr{H}}$ one obtains

$$
\begin{aligned}
\left(U_{f}(a, A) g\right)\left(\phi, \bar{\theta}_{1}, \ldots, \bar{\theta}_{N-1}\right)= & \exp \left[i a_{0} \cosh \phi\left(\sum_{i=1}^{N} \cosh \bar{\theta}_{i}\right)\right. \\
& \left.-i a_{1} \sinh \phi\left(\sum_{i=1}^{N} \cosh \bar{\theta}_{i}\right)\right] \cdot g\left(\phi-\alpha, \bar{\theta}_{1}, \ldots, \bar{\theta}_{N-1}\right),
\end{aligned}
$$

which corresponds to generators

$$
\begin{aligned}
& H_{f}=\cosh \phi\left(\sum_{i=1}^{N} \cosh \bar{\theta}_{i}\right) \\
& \mathbf{P}_{f}=\sinh \phi\left(\sum_{i=1}^{N} \cosh \bar{\theta}_{i}\right) \\
& L_{f}=i \dot{d}_{\phi} .
\end{aligned}
$$


Hence, if we set

$$
\begin{aligned}
H & =\cosh \phi\left(\sum_{i=1}^{N} \cosh \bar{\theta}_{i}+V\right), \\
\mathbf{P} & =\sinh \phi\left(\sum_{i=1}^{N} \cosh \bar{\theta}_{i}+V\right), \\
U(a, \Lambda) & =\exp (i a \cdot P) U_{f}(\Lambda),
\end{aligned}
$$

the $U(a, A)$ will also form a unitary positive energy representation provided $V$ is an operator on the fiber $L^{2}\left(R^{N-1} ; d \bar{\theta}_{1} \cdots d \bar{\theta}_{N-1}\right)$ such that the reduced Hamiltonian

$$
H_{r}=\sum_{i=1}^{N} \cosh \bar{\theta}_{i}+V
$$

is a positive operator. This will be the case for instance if one takes for $V$ a selfadjoint trace class operator that is positive, or small in norm. Moreover, in this case the Kato-Rosenblum theorem [22] implies that the reduced wave operators exist and are complete, so that the assumption made in Section 1 is not vacuous.

In anticipation of a more thorough discussion of $\mathrm{N}$-body interactions in Section 5 we would like to consider briefly a natural extension of (2.11) to the $N$-body case, viz., the interaction

$$
V=\sum_{1 \leqslant i<j \leqslant N} V_{i j}
$$

where

$$
\begin{aligned}
\left(V_{12} f\right)\left(\bar{\theta}_{1}, \ldots, \bar{\theta}_{N-1}\right)= & \int d \theta^{\prime} \hat{F}_{12}\left(\frac{1}{2}\left(\bar{\theta}_{1}-\bar{\theta}_{2}\right)-\theta^{\prime}\right) f\left(\frac{1}{2}\left(\bar{\theta}_{1}+\bar{\theta}_{2}\right)\right. \\
& \left.+\theta^{\prime}, \frac{1}{2}\left(\bar{\theta}_{1}+\bar{\theta}_{2}\right)-\theta^{\prime}, \ldots, \bar{\theta}_{N-1}\right)
\end{aligned}
$$

and where the other $V_{i j}$ are defined analogously. For the $\hat{F}_{i j}$ one could take for instance Fourier transforms of positive test functions so as to ensure positivity of $H_{\mathrm{r}}$. At first sight it would seem perfectly natural to refer to the $V_{i j}$ as "pair potentials." We shall do this indeed, but the name is less appropriate than it seems. To see why, let us consider the case that only $V_{12} \neq 0$. If one then transforms $H$ back to the $\theta_{1}, \ldots, \theta_{N}$ variables, the resulting interaction term depends on all $\theta_{i}$ because of the Jacobian factors and the $\cosh \phi$ factor in $H$. This already seems to show the difficulty. However, this fact is in a sense irrelevant. The point is that as soon as an interaction is present the concept of individual particle variables becomes obscure, a property the present scheme shares with relativistic field theories and the Bakamjian-Thomas framework. (In this connection we mention the "no-interaction" theorem (cf. Ref. [49] and references given there), showing no invariant world lines exist if an interaction is added in schemes of the type considered here.) What really matters is whether the 
$S$-operator corresponding to $V_{12}$ only depends on $\theta_{1}$ and $\theta_{2}$, since it is only asymptotically that the $\theta_{i}$ regain a clear meaning as individual particle variables. It is this property which is violated by $V_{12}$ : the factor $\cosh \bar{\theta}_{N}$ in (3.14) depends on all $\bar{\theta}_{1}, \ldots, \bar{\theta}_{N-1}$ and does not commute with $V_{12}$, so that the reduced $S$-operator will depend on all $\bar{\theta}_{i}$ (in general). Correspondingly, the $S$-operator will depend on all $\theta_{i}$. One can define a $V_{12}$ similar to (3.16) that does commute with $\cosh \bar{\theta}_{N}$ (cf. Section 5.2), in which case the reduced $S$-operator depends only on $\bar{\theta}_{1}, \bar{\theta}_{2}$. Although this implies that the $S$-operator commutes with $\theta_{3}, \ldots, \theta_{N}$, the fibers $S\left(\theta_{3}, \ldots, \theta_{N}\right)$ acting on $L^{2}\left(d \theta_{1} d \theta_{2}\right)$ will only be independent of $\theta_{3}, \ldots, \theta_{N}$ if the reduced $S$-operator does not depend on $\bar{\theta}_{1}+\bar{\theta}_{2}$. It is possible to introduce an interaction leading to such an $S$-operator, but only at the expense of relinquishing the linear form (3.14) for $H_{r}$. Since this is more transparent in terms of a momenta formulation, we defer a discussion of this to Section 5.2.

In the case of three space dimensions the difficulty signaled above makes it unlikely that one can define pair potentials for which the $S$-operator has the customary cluster properties [43], known to hold in nonrelativistic quantum mechanics [22, 44] and relativistic quantum field theory [45]. We shall come back to this in Section 5.2. However, in the case in hand, this difficulty is only mildly disturbing, since in one dimension the $S$-operator does not cluster in the usual sense, both in nonrelativistic quantum mechanics and in field theory. Since this simple fact does not seem to be widely known, it may be useful to include the easy proof: In nonrelativistic quantum mechanics the two-particle $S$-operator for a pair potential is given by (2.12), where the $\theta_{i}$ should be replaced by the particle momenta $k_{i}$. The usual cluster property, valid in three dimensions, states that

$$
\underset{s-\lim _{a \rightarrow \infty}}{ } U_{1}(\mathbf{a}) S U_{1}^{*}(\mathbf{a})=1,
$$

where $U_{1}(\mathbf{a})$ is translation of the first particle by a. In our case $U_{1}(a)$ is the multiplication operator $\exp \left(-i k_{1} a\right)$ so that

$$
\begin{aligned}
& \left(U_{1}(a) S U_{1}^{*}(a) f\right)\left(k_{1}, k_{2}\right) \\
& \quad=t\left(\frac{1}{2}\left(k_{1}-k_{2}\right)\right) f\left(k_{1}, k_{2}\right)+\exp \left[-i\left(k_{1}-k_{2}\right) a\right] r\left(\frac{1}{2}\left(k_{1}-k_{2}\right)\right) f\left(k_{2}, k_{1}\right) .
\end{aligned}
$$

In relativistic quantum field theory the two-particle $S$-operator below the production threshold is given by (2.12) so that one has as the analog of (3.18)

$$
\begin{aligned}
& \left(U_{1}(a) S U_{1}^{*}(a) f\right)\left(\theta_{1}, \theta_{2}\right) \\
& \quad=t\left(\frac{1}{2}\left(\theta_{1}-\theta_{2}\right)\right) f\left(\theta_{1}, \theta_{2}\right)+\exp \left[-i\left(p\left(\theta_{1}\right)-p\left(\theta_{2}\right)\right) a\right] r\left(\frac{1}{2}\left(\theta_{1}-\theta_{2}\right)\right) f\left(\theta_{2}, \theta_{1}\right),
\end{aligned}
$$

where $p(\theta)=m \sinh \theta$ is the momentum corresponding to rapidity $\theta$, and where $f=0$ if $\cosh \left(\frac{1}{2}\left(\theta_{1}-\theta_{2}\right)\right) \geqslant \frac{3}{2}$ (assuming for simplicity that the two particles are distinguishable and have the same mass $m$ ). It is clear that neither (3.18) nor (3.19) has a strong limit for $|a| \rightarrow \infty$. In physical terms this lack of clustering can be ascribed 
to the fact that in one dimension two particles cannot "mess" each other, even if they are first moved far apart; although their wave packets have spread by the time they start interacting, the interaction will take place over a longer time interval that makes up for the spreading. It should be emphasized that the clustering discussed here is very different from the factorization of the $S$-operator for the one-dimensional field theories discussed in the Introduction. Indeed, for these field theories it is clear from the same argument as given above that none of the scattering amplitudes clusters.

In closing this section we should like to repeat that the choice of $\bar{\theta}_{1}, \ldots, \bar{\theta}_{N}$ as internal variables has been made for convenience. Another rather natural choice would be to take $N-1$ difference variables $\theta_{i}-\theta_{j}$, since $H_{r}{ }^{0}$ can be written

$$
H_{r}^{0}=\left[\sum_{i, j-1}^{N} \cosh \left(\theta_{i}-\theta_{j}\right)\right]^{1 / 2} \text {. }
$$

Also, the interaction need not be introduced in a linear fashion as in (3.14). One could, e.g., add an integral operator acting on the difference variables under the square root in (3.20), the resulting operator $H_{r}$ being defined by the functional calculus as long as its square is positive. There is no difficulty in verifying that the arguments in Section 1.2 also hold true in this case. Moreover, by the invariance principle for the wave operators [22] the reduced $S$-operator for the pair $H_{r}{ }^{0}, H_{r}$ equals the reduced $S$-operator for the more tractable pair $\left(H_{r}^{0}\right)^{2},\left(H_{r}\right)^{2}$ (for suitable interactions).

\section{Two Spin-Zero Particles in Three Dimensions}

We shall now consider two spin-zero particles with masses $m_{1}, m_{2}>0$ in three space dimensions. The corresponding free representation of the Poincare group is given by unitary operators $U_{f}(a, A)$ on the Hilbert space $\mathscr{H}=L^{2}\left(R^{6} ; d \mathbf{p}_{1} d \mathbf{p}_{2}\right)$ as follows (cf. the Appendix):

$\left(U_{f}(a, \Lambda) f\right)\left(\mathbf{p}_{1}, \mathbf{p}_{2}\right)=\exp \left(i\left(p_{1}+p_{2}\right) \cdot a\right)\left(\frac{\left(\Lambda^{-1} p_{1}\right)_{0}\left(\Lambda^{-1} p_{2}\right)_{0}}{p_{1,0} p_{2,0}}\right)^{1 / 2} f\left(\overrightarrow{\Lambda^{-1} p_{1}}, \overrightarrow{\Lambda^{-1} p_{2}}\right)$.

Clearly, the generators of time resp. spacc translations are the multiplication operators

$$
\begin{aligned}
& H_{f}=E_{p_{1}}+E_{p_{2}}, \\
& \mathbf{P}_{f}=\mathbf{p}_{1}+\mathbf{p}_{2} .
\end{aligned}
$$

In order to change to center-of-mass variables we now introduce the 4-vectors

$$
k\left(\mathbf{p}_{1}, \mathbf{p}_{2}\right)=\left(p_{1}+p_{2}\right) / M,
$$


where $M$ is the invariant mass

$$
M^{2} \equiv\left(p_{1}+p_{2}\right)^{2}
$$

and

$$
q_{i}\left(\mathbf{p}_{1}, \mathbf{p}_{2}\right)=B\left(\frac{p_{1}+p_{2}}{M}\right)^{-1} p_{i}, \quad i=1,2
$$

Thus, $q_{i}$ is the 4-momentum of the $i$ th particle in the center-of-mass frame defined by the pure boost $\left(p_{1}+p_{2}\right) / M \rightarrow(1,0)$, (cf. (A10) and (A11)) and we can set

$$
\left.\left.\underset{2}{q_{1}}=\left(\left[|\mathbf{q}|^{2}+\underset{2}{\left(m_{1}\right.}\right)^{2}\right]^{1 / 2},\right\lrcorner_{-} \mathbf{q}\right)
$$

Hence, we may regard $p_{1}$ and $p_{2}$ as functions of $\mathbf{k}$ and $\mathbf{q}$ :

$$
\begin{aligned}
& p_{1}(\mathbf{k}, \mathbf{q})=B(k) q_{\mathbf{1}} \\
& p_{2}(\mathbf{k}, \mathbf{q})=B(k) q_{2} .
\end{aligned}
$$

It easily follows that the transformation $\left(\mathbf{p}_{1}, \mathbf{p}_{2}\right) \rightarrow(\mathbf{k}, \mathbf{q})$ is a diffeomorphism of $R^{6}$ onto itself.

We now map $\mathscr{H}$ isometrically onto $\overline{\mathscr{H}}=L^{2}(d \mathbf{k} d \mathbf{q})$ by setting

$$
(I f)(\mathbf{k}, \mathbf{q}) \equiv J(\mathbf{k}, \mathbf{q})^{\mathbf{1 / 2}} f\left(\mathbf{p}_{\mathbf{1}}(\mathbf{k}, \mathbf{q}), \mathbf{p}_{\mathbf{2}}(\mathbf{k}, \mathbf{q})\right)
$$

Here, $J$ is the Jacobian of the transformation:

$$
J(\mathbf{k}, \mathbf{q}) \equiv \frac{\partial\left(\mathbf{p}_{1}, \mathbf{p}_{2}\right)}{\partial(\mathbf{k}, \mathbf{q})}=\frac{\left(E_{q_{1}}+E_{q_{2}}\right)^{3}}{k_{0}} \frac{\left(I_{r} k \cdot q_{1}\right)}{E_{q_{1}}} \frac{\left(I_{r} k \cdot q_{2}\right)}{E_{q_{2}}}
$$

(cf. (A14)). We then have, using (A13),

$$
\left(I^{-1} g\right)\left(\mathbf{p}_{1}, \mathbf{p}_{2}\right)=\tilde{J}\left(\mathbf{p}_{1}, \mathbf{p}_{2}\right)^{-1 / 2} g\left(\mathbf{k}\left(\mathbf{p}_{1}, \mathbf{p}_{2}\right), \mathbf{q}\left(\mathbf{p}_{1}, \mathbf{p}_{2}\right)\right)
$$

where

$$
\tilde{J}\left(\mathbf{p}_{1}, \mathbf{p}_{2}\right)-\frac{M^{6}}{\left(m_{1}^{2}+p_{1} \cdot p_{2}\right)\left(m_{2}^{2}+p_{1} \cdot p_{2}\right)} \cdot \frac{E_{p_{1}} E_{p_{2}}}{E_{p_{1}}+E_{p_{2}}} .
$$

A straightforward calculation now shows that the action of $U_{f}(a, \Lambda)$ on $\overline{\mathscr{P}}$ is given by $\left(U_{f}(a, \Lambda) g\right)(\mathbf{k}, \mathbf{q})=\exp \left[i\left(E_{\mathbf{q}_{1}}+E_{q_{2}}\right) k \cdot a\right]\left(\frac{\left(\Lambda^{-1} k\right)_{0}}{k_{0}}\right)^{1 / 2} g\left(\overrightarrow{\Lambda^{-1}} k, R(k, \Lambda) \mathbf{q}\right)$

(As before, $U_{f}$ denotes here the operator $I U_{f} I^{-1}$ on $\overline{\mathscr{H}}$. To verify (4.12), use (A13) and (A17).) 
Clearly,

$$
\begin{aligned}
& H_{f}=k_{0}\left(E_{a_{1}}+E_{q_{2}}\right), \\
& \mathbf{P}_{f}=\mathbf{k}\left(E_{q_{1}}+E_{q_{2}}\right),
\end{aligned}
$$

showing the desired product form.

Now define

$$
(V f)(\mathbf{k}, \mathbf{q})=\int d \mathbf{q}^{\prime} K\left(\mathbf{q}, \mathbf{q}^{\prime}\right) f\left(\mathbf{k}, \mathbf{q}^{\prime}\right)
$$

A calculation using (4.12) and (A18) shows that $V$ commutes with $U_{f}(\Lambda)$ provided $K$ is a function of the inner products $\mathbf{q} \cdot \mathbf{q}, \mathbf{q} \cdot \mathbf{q}^{\prime}$, and $\mathbf{q}^{\prime} \cdot \mathbf{q}^{\prime}$ only. (In particular an operator of the form

$$
\left(V_{\phi} f\right)(\mathbf{k}, \mathbf{q})=\int d \mathbf{q}^{\prime} \hat{\phi}\left(\mathbf{q}-\mathbf{q}^{\prime}\right) f\left(\mathbf{k}, \mathbf{q}^{\prime}\right),
$$

with $\phi$ spherically symmetric, has this property.) Assuming this from now on we set

$$
\begin{aligned}
H & =k_{0}\left(E_{a_{1}}+E_{a_{2}}+V\right), \\
\mathbf{P} & =\mathbf{k}\left(E_{a_{1}}+E_{a_{2}}+V\right), \\
U(a, \Lambda) & =\exp (\text { ia } \cdot P) U_{f}(\Lambda) .
\end{aligned}
$$

It follows as described in Section 1.1 that $U(a, A)$ is a unitary positive energy representation, provided $K$ is such that the reduced Hamiltonian

$$
H_{r} \equiv E_{q_{1}}+E_{q_{2}}+V
$$

defines a positive operator on the fiber $L^{2}(d \mathbf{q})$. If the wave operators exist, they will intertwine the free and interacting representations, and the corresponding $S$-operator will commute with $U_{f}(a, \Lambda)$. We expect that a unitary $S$-operator exists satisfying

$$
\begin{aligned}
(S f)(\mathbf{k}, \mathbf{q})= & f(\mathbf{k}, \mathbf{q})-2 \pi i \int d \mathbf{q}^{\prime} \delta\left(E_{a_{1}}+E_{a_{\mathbf{2}}}-E_{a_{1}^{\prime}}-E_{a_{2}^{\prime}}\right) \\
& \times T\left(E_{q_{1}}+E_{q_{2}},\left|\mathbf{q}-\mathbf{q}^{\prime}\right|^{2}\right) g\left(\mathbf{k}, \mathbf{q}^{\prime}\right)
\end{aligned}
$$

for a large class of "reasonable" potentials, containing at least those $V_{\phi}$ for which $\phi$ is smooth and rapidly decaying (cf. (4.15)). Equivalently, on $\mathscr{H}$ one should have

$$
\begin{aligned}
(S f)\left(\mathbf{p}_{1}, \mathbf{p}_{2}\right)= & f\left(\mathbf{p}_{1}, \mathbf{p}_{2}\right)-2 \pi i\left(E_{p_{1}} E_{p_{2}}\right)^{-1 / 2} \int d \mathbf{p}_{1}^{\prime} d \mathbf{p}_{2}^{\prime} \delta\left(p_{1}+p_{2}-p_{1}^{\prime}-p_{2}^{\prime}\right) \\
& \times A(s, t)\left(E_{p_{1}} E_{p_{2}^{\prime}}\right)^{-1 / 2} f\left(\mathbf{p}_{1}^{\prime}, \mathbf{p}_{2}^{\prime}\right),
\end{aligned}
$$


where

$$
\begin{gathered}
s=\left(p_{1}+p_{2}\right)^{2}, \\
t=\left(p_{1}-p_{1}^{\prime}\right)^{2}
\end{gathered}
$$

are the customary Mandelstam variables, and

$$
A(s, t) \equiv \frac{\left[s^{2}-\left(m_{1}^{2}-m_{2}^{2}\right)^{2}\right]}{4 s} T\left(s^{1 / 2},-t\right) .
$$

We close this section with some remarks:

(i) (Bound states) Since $H_{r}$ commutes with rotations on $L^{2}(d \mathbf{q})$, one may classify its bound states according to their total angular momentum. If $\left\{f_{i}(\mathbf{q})\right\}_{i=-l}^{l}$ is a set of bound states with energy $m$ transforming into each other under rotations, then it is easy to see that the restriction of $U(a, A)$ to the tensor product of $L^{2}(d \mathbf{k})$ and the span of the $f_{i}(\mathbf{q})$ is an irreducible representation of mass $m$ and spin $l$.

(ii) (Nonrelativistic limit) If one chooses $V=V_{\phi}$ as in (4.15), then the nonrelativistic limit of $H$ is unitarily equivalent to the Schrödinger operator

$$
H_{\mathrm{nr}}=m_{1}+m_{2}-\frac{1}{2 m_{1}} \Delta_{1}-\frac{1}{2 m_{2}} \Delta_{2}+\phi\left(\mathbf{x}_{1}-\mathbf{x}_{2}\right)
$$

on $L^{2}\left(d \mathbf{x}_{1} d \mathbf{x}_{2}\right)$, where

$$
\phi(\mathbf{x}) \equiv \int d \mathbf{p} \exp (i \mathbf{p} \cdot \mathbf{x}) \hat{\phi}(\mathbf{p})
$$

To see this, Fourier transform $H_{\mathrm{nr}}$ to $L^{2}\left(d \mathbf{p}_{1} d \mathbf{p}_{2}\right)$ and then to $\overline{\mathscr{P}} \equiv L^{2}(d \mathbf{k} d \mathbf{q})$, where

$$
\begin{aligned}
& \mathbf{k} \equiv\left(\mathbf{p}_{1}+\mathbf{p}_{2}\right) /\left(m_{1}+m_{2}\right), \\
& \mathbf{q} \equiv\left(m_{2} \mathbf{p}_{1}-m_{1} \mathbf{p}_{2}\right) /\left(m_{1}+m_{2}\right)
\end{aligned}
$$

On $\overrightarrow{\mathscr{H}}, H_{\mathrm{nr}}$ acts as

$$
\begin{aligned}
\left(H_{\mathrm{nr}} f\right)(\mathbf{k}, \mathbf{q})= & {\left[\left(m_{\mathbf{1}}+m_{2}\right)\left(1+\frac{1}{2}|\mathbf{k}|^{2}\right)+\frac{1}{2}|\mathbf{q}|^{2}\left(\frac{1}{m_{1}}+\frac{1}{m_{2}}\right)\right] f(\mathbf{k}, \mathbf{q}) } \\
& +\int d \mathbf{q}^{\prime} \hat{\phi}\left(\mathbf{q}-\mathbf{q}^{\prime}\right) f\left(\mathbf{k}, \mathbf{q}^{\prime}\right)
\end{aligned}
$$

which is indeed the nonrelativistic limit of $H$.

(iii) (Clustering) If one chooses $V=V_{\phi}$ with $\phi$ a bounded function in $L^{\mathbf{1}}(d \mathbf{x})$, it follows by adapting the proof of Theorem XI 30 in Ref. [22] that the reduced wave operators $\tilde{W}_{ \pm}$exist and are complete. As a result the $S$-operator $S=W_{+}^{*} W_{-}$is unitary. It is easy to see that $S$ has the cluster property, i.e., that on $\overline{\mathscr{H}}$

$$
s-\lim _{|\mathbf{a}| \rightarrow \infty} U_{1}(\mathbf{a}) S U_{1}^{*}(\mathbf{a})=1,
$$


where $U_{1}(\mathbf{a})$ is the multiplication operator $\exp \left(-\overrightarrow{i B(k) q_{1}} \cdot\right.$ a) on $\overline{\mathscr{H}}$. Indeed, using (A11) and the fact that $S$ commutes with $E_{q_{1}}$ and $k$ it follows that (4.26) is implied by

$$
\operatorname{sim}_{|\mathbf{a}| \rightarrow \infty} U(\mathbf{a}) \tilde{W}_{ \pm} U^{*}(\mathbf{a})=1,
$$

on $L^{2}(d \mathbf{q})$, where $U(\mathbf{a})$ is the multiplication operator $\exp (-\mathbf{i q} \cdot \mathbf{a})$. However, (4.27) readily follows by adapting the proof of Theorem XI 33 in Ref. [22].

(iv) (A curiosity) As mentioned before, the linear form (4.16) is only the most obvious choice to introduce the interaction. We close this section by pointing out an amusing alternative in case $m_{1}=m_{2} \equiv m$. Consider the operator

$$
H_{s}=|\mathbf{q}|^{2}+V_{\phi} \text {. }
$$

If we choose $\phi$ such that $H_{s}>-m^{2}$ we may set

$$
\begin{aligned}
P & =2 k\left[H_{s}+m^{2}\right]^{1 / 2}, \\
U(a, \Lambda) & =\exp (i a \cdot P) U_{f}(\Lambda) .
\end{aligned}
$$

Arguing as in Section 1.1 one readily verifies that $U(a, \Lambda)$ is a unitary positive energy representation and that the $S$-operator is Lorentz invariant. This choice is of some interest since $H_{s}$ is just the nonrelativistic Schrödinger operator for a particle of mass $\frac{1}{2}$ in the central potential $\phi$. For suitable $\phi$ the invariance principle for the wave operators [22] is easily seen to imply that the nonrelativistic and relativistic $S$-operators are the same on $L^{2}(d q)$. Hence, any nonrelativistic $S$-matrix corresponding to a particle in a central potential $\phi$ can also arise in a relativistic framework for a large class of $\phi$, viz., those for which $H_{s}-\mid m^{2}>0$ and for which the invariance principle applies.

\section{5. $N$ Particles in Three Dimensions with Arbitrary Sins and Masses}

In this final section the general case of $N$ particles with arbitrary masses $m_{i}>0$ and spins $s_{i}$ is studied $(i=1, \ldots, N)$. We have divided the subject matter into several subsections. As before, we begin by filling in the details of the construction in Section 1.1 and then consider several applications.

\subsection{Formalism}

The particles can be described by wavefunctions from the Hilbert space $\mathscr{H}=$ $L^{2}\left(R^{3 N} ; d \mathbf{p}_{1} \cdots d \mathbf{p}_{N}\right)^{\left(2 s_{1}+1\right) \cdots\left(2 s_{N}+1\right)}$. On $\mathscr{H}$ the free representation of the Poincare group reads (cf. the Appendix)

$$
\begin{aligned}
\left(U_{f}(a, \Lambda) f\right)_{\alpha}\left(\mathbf{p}_{1}, \ldots, \mathbf{p}_{N}\right)= & \exp \left(i a \cdot \sum_{i=1}^{N} p_{i}\right) \prod_{i=1}^{N}\left(\frac{\left(\Lambda^{-1} p_{i}\right)_{0}}{p_{i, 0}}\right)^{1 / 2} \\
& \times U^{s_{i}}\left[\frac{p_{i}}{m_{i}}, A\right]_{\alpha_{i} B_{i}}^{*} f_{\beta}\left(\overrightarrow{\Lambda^{-1} p_{1}}, \ldots, \overrightarrow{\Lambda^{-1} p_{N}}\right)
\end{aligned}
$$


where $\alpha_{i}, \beta_{i}=1, \ldots, 2 s_{i}+1$. Hence,

$$
\begin{aligned}
& H_{f}=\sum_{i=1}^{N} E_{p_{i}}, \\
& \mathbf{P}_{f}=\sum_{i=1}^{N} \mathbf{p}_{i} .
\end{aligned}
$$

Proceeding as in Section 4 we set

$$
\begin{aligned}
k\left(\mathbf{p}_{1}, \ldots, \mathbf{p}_{N}\right) & =\sum_{i=1}^{N} p_{i} / M, \\
M^{2} & \equiv\left(\sum_{i=1}^{N} p_{i}\right)^{2},
\end{aligned}
$$

and

$$
q_{j}\left(\mathbf{p}_{1}, \ldots, \mathbf{p}_{N}\right)=B\left(\sum_{i=1}^{N} p_{i} / M\right)^{-1} p_{j}, \quad j=1, \ldots, N .
$$

Thus, the $q_{i}$ are the 4-momenta of the particles in the center-of-mass frame defined by the pure boost $\sum p_{i} / M \rightarrow(1, \mathbf{0})$. We shall choose $\mathbf{k}, \mathbf{q}_{\mathrm{l}}, \ldots, \mathbf{q}_{N-1}$ as the independent variables. The inverse transformation then reads

$$
p_{i}\left(\mathbf{k}, \mathbf{q}_{1}, \ldots, \mathbf{q}_{N-1}\right)=B(k) q_{i}, \quad i=1, \ldots, N,
$$

where

$$
\begin{gathered}
k_{0}=\left(|\mathbf{k}|^{2}+1\right)^{1 / 2} \\
q_{i, 0}-\left(\left|\mathbf{q}_{i}\right|^{2}+m_{i}^{2}\right)^{1 / 2}, \quad i=1, \ldots, N
\end{gathered}
$$

and

$$
\mathbf{q}_{N}=-\sum_{i=1}^{N-1} \mathbf{q}_{i}
$$

Again it is clear that the transformation $\left(\mathbf{p}_{1}, \ldots, \mathbf{p}_{N}\right) \rightarrow\left(\mathbf{k}, \mathbf{q}_{1}, \ldots, \mathbf{q}_{N-1}\right)$ is a diffeomorphism of $R^{3 N}$. Hence, we can define unitary operators

$$
\begin{aligned}
(I f)\left(\mathbf{k}, \mathbf{q}_{1}, \ldots, \mathbf{q}_{N-1}\right) & =J^{1 / 2} f\left(\mathbf{p}_{1}\left(\mathbf{k}, \mathbf{q}_{1}, \ldots, \mathbf{q}_{N-1}\right), \ldots, \mathbf{p}_{N}\left(\mathbf{k}, \mathbf{q}_{1}, \ldots, \mathbf{q}_{N-1}\right)\right), \\
\left(I^{-1} g\right)\left(\mathbf{p}_{1}, \ldots, \mathbf{p}_{N}\right) & =\tilde{J}^{-1 / 2} g\left(\mathbf{k}\left(\mathbf{p}_{1}, \ldots, \mathbf{p}_{N}\right), \mathbf{q}_{1}\left(\mathbf{p}_{1}, \ldots, \mathbf{p}_{N}\right), \ldots, \mathbf{q}_{N-1}\left(\mathbf{p}_{1}, \ldots, \mathbf{p}_{N}\right)\right)
\end{aligned}
$$

mapping $\mathscr{H}$ onto $\overline{\mathscr{H}} \equiv L^{2}\left(R^{3 N} ; d \mathbf{k} d \mathbf{q}_{1} \cdots d \mathbf{q}_{N-1}\right)$ resp. $\mathscr{\mathscr { H }}$ onto $\mathscr{H}$. Here, $J$ is the Jacobian of the transformation in terms of $\mathbf{k}, \mathbf{q}_{1}, \ldots, \mathbf{q}_{N-1}$, while $\tilde{J}$ is the Jacobian as a function of $\mathbf{p}_{1}, \ldots, \mathbf{p}_{N}$. Explicitly one finds

$$
J=\frac{\left(\sum_{j=1}^{N} E_{q_{j}}\right)^{3}}{k_{0}} \prod_{i=1}^{N} \frac{\left(I_{r} k \cdot q_{i}\right)}{E_{q_{i}}},
$$


so that using (A13) one obtains

$$
\tilde{J}=M^{4+N} \prod_{i=1}^{N}\left(p_{i} \cdot \sum_{j=1}^{N} p_{j}\right)^{-1} E_{3_{i}} / \sum_{j=1}^{N} E_{2 j_{j}} .
$$

On $\mathscr{\mathscr { H }}$ we then get

$$
\begin{aligned}
& H_{f}==k_{0} \sum_{i=1}^{N} E_{q_{i}}, \\
& \mathbf{P}_{f}=\mathbf{k} \sum_{i=1}^{N} E_{a_{i}},
\end{aligned}
$$

and, more generally,

$$
\begin{aligned}
\left(U_{f}(a, \Lambda) g\right)_{\alpha}\left(\mathbf{k}, \mathbf{q}_{\mathbf{1}}, \ldots, \mathbf{q}_{N-1}\right) \\
=\exp \left[i a \cdot k\left(\sum_{j=1}^{N} E_{a_{j}}\right)\right]\left(\frac{\left(A^{-1} k\right)_{0}}{k_{0}}\right)^{1 / 2} \prod_{i=1}^{N} U^{s_{i}}\left[B(k) q_{i} / m_{i}, A\right]_{\alpha_{i} B_{i}}^{*} \\
\quad \times g_{\beta}\left(\overrightarrow{\Lambda^{-1} k}, R(k, \Lambda) \mathbf{q}_{\mathbf{1}}, \ldots, R(k, \Lambda) \mathbf{q}_{N-1}\right) .
\end{aligned}
$$

In accordance with Section 1.1 we now set

$$
P=k H_{r}
$$

where

$$
H_{r}=\sum_{i=1}^{N} E_{q_{i}}+V
$$

Here, $V$ is such that $H_{r}$ is positive, and moreover

$$
\left[U_{f}(\Lambda), V\right]=0
$$

Then an easy extension of the arguments in Section 1.1 shows that

$$
U(a, \Lambda)=\exp (i a \cdot P) U_{f}(\Lambda)
$$

defines a new positive energy representation of the Poincare group, and that the $S$-operator is Lorentz invariant if $V$ is decomposable w.r.t. the direct integral $\overline{\mathscr{H}} \simeq$ $\int_{R^{3}}^{\oplus} d \mathbf{k} \tilde{\mathscr{H}}$. This is equivalent to $V$ acting like

$$
(V(\phi \otimes \psi))_{\alpha}\left(\mathbf{k}, \mathbf{q}_{1}, \ldots, \mathbf{q}_{N-1}\right)=\phi(\mathbf{k})(V(\mathbf{k}) \psi)_{\boldsymbol{a}}\left(\mathbf{q}_{1}, \ldots, \mathbf{q}_{N-1}\right)
$$


on vectors $\phi \otimes \psi \in \overline{\mathscr{H}}$, where $\phi \in \mathscr{H}_{\mathbf{C M}} \equiv L^{2}(d \mathbf{k})$ and $\psi \in \widetilde{\mathscr{H}} \equiv L^{2}\left(R^{3 N-3} ; d \mathbf{q}_{1} \cdots\right.$ $\left.d \mathbf{q}_{N-1}\right)^{\left(2 s_{1}+1\right) \cdots\left(2 s_{N}+1\right)}$. A large class of interactions satisfying (5.15) and (5.17) may be found by setting

$$
\begin{aligned}
(V f)_{\mathbf{\alpha}}\left(\mathbf{k}, \mathbf{q}_{1}, \ldots, \mathbf{q}_{N-1}\right)= & \sum_{l=1}^{L} \int_{1} d \mathbf{q}_{1}^{\prime} \cdots d \mathbf{q}_{N-1}^{\prime} K_{l}\left(\mathbf{q}_{1}, \ldots, \mathbf{q}_{N-1} ; \mathbf{q}_{1}^{\prime}, \ldots, \mathbf{q}_{N-1}^{\prime}\right) \\
& \times \prod_{i-1}^{N} \mathscr{G}_{l}^{s_{i}}\left(k, q_{i}, q_{i}^{\prime}\right)_{\alpha_{i} \beta_{i}} f_{\beta}\left(\mathbf{k}, \mathbf{q}_{\mathbf{1}}^{\prime}, \ldots, \mathbf{q}_{N-1}^{\prime}\right),
\end{aligned}
$$

where the $K_{l}$ are generalized functions and the $\mathscr{G}_{l}^{s^{i}}\left(2 s_{i}+1\right) \times\left(2 s_{i}+1\right)$ matrix functions. It is clear that (5.17) is satisfied, while a straightforward analysis using several relations from the Appendix shows that (5.15) is satisfied if the $K_{l}$ are invariant under any rotation of the $\mathbf{q}_{i}$ and $\mathbf{q}_{i}^{\prime}$, and if the $\mathscr{G}_{i}^{s_{i}}$ are of the form

$$
\mathscr{G}^{s}\left(k, q, q^{\prime}\right)=U^{s}[q / m, \hat{k}] G^{s}\left(q, q^{\prime}\right) U^{s}\left[q^{\prime} / m, \hat{k}\right]^{*},
$$

where $G^{s}$ is a $(2 s+1) \times(2 s+1)$ matrix function satisfying

$$
\mathscr{L}^{s}[A] G^{s}\left(q, q^{\prime}\right) \mathscr{H}^{s}\left[A^{*}\right]=G^{s}\left(\Lambda(A) q, \Lambda(A) q^{\prime}\right), \quad \forall A \in S U(2) .
$$

(This is sufficient, but not necessary to satisfy (5.15). For instance, from (A23) it follows that operators containing terms like $\mathbf{S}_{i} \cdot \mathbf{S}_{j}$ are also allowed. However, we have no occasion to consider such operators.) Note that

$$
G^{s}\left(q, q^{\prime}\right)=\mathscr{G}^{s}\left((1, \mathbf{0}), q, q^{\prime}\right)
$$

and that $\mathscr{G}^{\mathrm{s}}$ is a self-adjoint integral kernel if $G^{\mathrm{s}}$ is, i.e., if

$$
G^{s}\left(q, q^{\prime}\right)=G^{s}\left(q^{\prime}, q\right)^{*}
$$

Assuming from now on that (5.20) and (5.22) are satisfied and that the integral kernels $K_{l}$ are invariant under rotations and self-adjoint as well, i.e.,

$K_{l}\left(\mathbf{q}_{1}, \ldots, \mathbf{q}_{N-1} ; \mathbf{q}_{1}^{\prime}, \ldots, \mathbf{q}_{N-1}^{\prime}\right)=\bar{K}_{l}\left(\mathbf{q}_{1}^{\prime}, \ldots, \mathbf{q}_{N-1}^{\prime} ; \mathbf{q}_{1}, \ldots, \mathbf{q}_{N-1}\right), \quad l=1, \ldots, L$,

it is clear that $V$ is formally self-adjoint. Note that it follows from (5.19) that the existence of the reduced wave operators for $\mathbf{k}=0$ implies their existence for any $\mathbf{k}$ and thus, by dominated convergence, the existence of the wave operators.

There is evidently a great amount of freedom in choosing the $K_{l}$ and $G_{l}{ }^{\circ}$. In particular, as in Section 3 onc could easily choose them such that $V(\mathbf{0})$ is a positive trace class operator, in which case the wave operators exist and are complete. However, we should like to conclude this paper by discussing interactions that seem more interesting to us. 


\subsection{Pair Potentials and Their Scattering Theory}

In this subsection we assume that all particles have spin zero. A natural $N$-particle extension of the eminently "reasonable" pair potential $V_{b}$ from Section 4 (cf. (4.15)) would then be to define

$$
V=\sum_{1 \leqslant i<j \leqslant N} V_{i j}
$$

where

$$
\begin{aligned}
\left(V_{12} g\right)\left(\mathbf{q}_{1}, \mathbf{q}_{2}, \ldots, \mathbf{q}_{N-1}\right)= & \int d \mathbf{q}^{\prime} \hat{\phi}_{12}\left(\frac{1}{2}\left(\mathbf{q}_{1}-\mathbf{q}_{2}\right)-\mathbf{q}^{\prime}\right) \\
& \cdot g\left(\frac{1}{2}\left(\mathbf{q}_{1}+\mathbf{q}_{2}\right)+\mathbf{q}^{\prime}, \frac{1}{2}\left(\mathbf{q}_{1}+\mathbf{q}_{2}\right)-\mathbf{q}^{\prime}, \ldots, \mathbf{q}_{N-1}\right)
\end{aligned}
$$

and where the potential $V_{i j}$ is defined such that it would take the same form as $V_{12}$ under the transformation $\mathbf{q}_{1}, \mathbf{q}_{2}, \ldots, \mathbf{q}_{i}, \ldots, \mathbf{q}_{j}, \ldots, \mathbf{q}_{N} \rightarrow \mathbf{q}_{i}, \mathbf{q}_{j}, \ldots, \mathbf{q}_{1}, \ldots, \mathbf{q}_{2}, \ldots, \mathbf{q}_{N}$. In particular, this implies that $V_{1 N}$ is given by

$$
\left(V_{1 N} g\right)\left(\mathbf{q}_{1}, \mathbf{q}_{2}, \ldots, \mathbf{q}_{N-1}\right)=\int d \mathbf{q}^{\prime} \hat{\phi}_{1 N}\left(\mathbf{q}_{1}-\mathbf{q}^{\prime}\right) g\left(\mathbf{q}^{\prime}, \mathbf{q}_{2}, \ldots, \mathbf{q}_{N-1}\right) .
$$

(Note that the interaction (5.24) is of the form (5.18) with $L=1$ if suitable $\delta$-functions are included in $K_{1}$ and if the $G^{0}$ are set equal to one.) Of course, the $\phi_{i j}$ are assumed to be spherically symmetric to ensure Lorentz invariance, and such that $H_{r}{ }^{0}+V$ is positive. This choice of interaction may be considered as a generalization of the pair potentials of nonrelativistic $N$-body Schrödinger theory to the relativistic framework considered here, since the nonrelativistic limit of $H$ is unitarily equivalent to the Schrödinger operator

$$
H_{\mathrm{nr}}=\sum_{i=1}^{N} m_{i}-\sum_{i=1}^{N} \Delta_{i} / 2 m_{i}+\sum_{i<j} \phi_{i j}\left(\mathbf{x}_{i}-\mathbf{x}_{j}\right)
$$

on $L^{2}\left(d \mathbf{x}_{1} \cdots d \mathbf{x}_{N}\right)$. Indeed, Fourier transformation of $H_{\mathrm{nr}}$ to $L^{2}\left(d \mathbf{p}_{1} \cdots d \mathbf{p}_{N}\right)$ and the subsequent transformation

$$
\begin{gathered}
\mathbf{k}=\sum_{i=1}^{N} \mathbf{p}_{i} / \sum_{i=1}^{N} m_{i}, \\
\mathbf{q}_{i}=\mathbf{p}_{i}-m_{i} \mathbf{k}, \quad i=1, \ldots, N
\end{gathered}
$$

to $\overline{\mathscr{H}} \equiv L^{2}\left(d \mathbf{k} d \mathbf{q}_{1} \cdots d \mathbf{q}_{N-1}\right)$ brings $H_{\mathrm{nr}}$ to the form

$$
H_{\mathrm{nr}}=\left(\sum_{i=1}^{N} m_{i}\right)\left(1+\frac{1}{2}|\mathbf{k}|^{2}\right)+\sum_{i=1}^{N}\left|\mathbf{q}_{i}\right|^{2} / 2 m_{i}+\sum_{i<j} V_{i j},
$$


which is clearly the nonrelativistic limit of $H$. (We mention in passing that after this transformation the Galileil invariance of the nonrelativistic $S$-operator for spherically symmetric pair potentials becomes obvious. Indeed, in these coordinates the Galilei boost generator $\sum_{i=1}^{N} m_{i} \mathbf{x}_{i}$ becomes the operator $i \partial_{\mathrm{k}}$.)

It can be proved by a stationary phase analysis (using the equation

$$
\mathbf{v}(\mathbf{k} \cdot \mathbf{a})+\mathbf{a}+\mathbf{k}(\mathbf{k} \cdot \mathbf{a}) /\left(k_{0}+1\right) \neq 0, \quad|\mathbf{v}|<1, \quad \mathbf{a} \neq 0
$$

and Theorem XI.14 in Ref. [22]) that $H$ clusters in the sense that on $\overline{\mathscr{H}}$

$$
\operatorname{sim}_{|\mathbf{a}| \rightarrow \infty} U_{i}(\mathbf{a}) V_{i j} U_{i}^{*}(\mathbf{a})=0, \quad 1 \leqslant i<j \leqslant N,
$$

where $U_{i}(\mathbf{a})$ is the multiplication operator $\exp \left(-i \overrightarrow{B(k) q_{i}} \cdot \mathbf{a}\right)$ on $\overrightarrow{\mathscr{H}}$, provided the potentials $\phi_{i j}$ are in $C_{0}{ }^{\infty}$ (this condition may be relaxed). Hence, a natural definition of cluster decomposition Hamiltonians would seem to be to set the intercluster potentials in $H_{r}$ equal to zero. The resulting operator is then a sum of mutually commuting cluster Hamiltonians (with unremoved centers of mass), as in Schrödinger theory. However, because of the nonquadratic dependence of $\left(|\mathbf{q}|^{2}+m^{2}\right)^{1 / 2}$ on $\mathbf{q}$ one cannot factor out the kinetic energy of the center of mass of two clusters as in the Schrödinger case, so that it seems quite hard to show that the clustered channel wave operators exist, even for $\phi_{i j} \in C_{0}{ }^{\infty}$. (Except for the channel in which all particles are free: in this case the required decay of $\left\|V_{i j} \exp \left(-i H_{r}{ }^{0} t\right) f\right\|$ can be proved by a stationary phase argument for the dense set of $f \in C_{0}{ }^{\infty}$ whose support is disjoint from the hypersurfaces $\mathbf{q}_{i} / E_{q_{i}}-\mathbf{q}_{j} / E_{q_{j}}=0$, where $1 \leqslant i<j \leqslant N$; again, the condition $\phi_{i j} \in C_{0}^{\infty}$ can be relaxed.) Even if this could be shown, the next step (cf. Ref. [22]), viz., the definition of channels, channel Hilbert space, etc., is far from clear. (Notice that these definitions will be unwieldy even in the Schrödinger case, if the coordinates (5.28) are used.) The latter difficulty does not arise if one defines the cluster decomposition Hamiltonians by making the change of coordinates (5.2), (5.3) for each cluster separately and by then adding the cluster potentials (note that the two definitions coincide in the Schrödinger case). The definition of channel, etc., could then proceed as in Schrödinger theory. However, in this case the existence of the clustered channel wave operators will be very hard to prove (or disprove) since several nonlinear coordinate changes are involved.

There are other more physical problems with this type of interaction. Although our notation and the result (5.31) may look very suggestive, the physical interpretation of the $U_{i}(\mathbf{a})$ as translations of the $i$ th particle and of the $V_{i j}$ as pair potentials is dubious. Indeed, the translation of the whole system by a is not equal to $\prod_{i=1}^{N} U_{i}(\mathbf{a})$ since we have modified $\mathbf{P}_{f}$, while it is unlikely that if, e.g., only $\phi_{12} \neq 0$, the $S$-operator will be independent of $\mathbf{p}_{3}, \ldots, \mathbf{p}_{N}$ (although it will not change them; cf. at this point the discussion in Section 3). This would clearly imply it does not have the usual cluster properties. It is therefore of interest to ask whether one can define a reduced Hamiltonian such that $S$ is manifestly independent of $\mathbf{p}_{3}, \ldots, \mathbf{p}_{N}$. Since $S$ is Lorentz invariant, this means it should have the form (4.19) in its action on $\mathbf{p}_{\mathbf{1}}, \mathbf{p}_{\mathbf{2}}$. If one 
transforms this to $\overline{\mathscr{H}}$ one finds that $S$ is independent of $\mathbf{k}, \mathbf{q}_{3}, \ldots, \mathbf{q}_{N-1}$, and acts exactly like (4.19) on $\mathbf{q}_{1}, \mathbf{q}_{2}$, i.e., one has

$$
\begin{aligned}
(S f)\left(\mathbf{k}, \mathbf{q}_{1}, \mathbf{q}_{2}, \ldots, \mathbf{q}_{N-1}\right) & \\
= & f\left(\mathbf{k}, \mathbf{q}_{1}, \mathbf{q}_{2}, \ldots, \mathbf{q}_{N-1}\right)-2 \pi i\left(E_{\alpha_{1}} E_{q_{2}}\right)^{-1 / 2} \int d \mathbf{q}_{1}^{\prime} d \mathbf{q}_{2}^{\prime} \delta\left(q_{1}+q_{2}-q_{1}^{\prime}-q_{2}^{\prime}\right) \\
& \times A\left(\left(q_{1}+q_{2}\right)^{2},\left(q_{1}-q_{1}^{\prime}\right)^{2}\right)\left(E_{q_{1}} E_{\alpha_{2}^{\prime}}\right)^{-1 / 2} f\left(\mathbf{k}, \mathbf{q}_{1}^{\prime}, \mathbf{q}_{2}^{\prime}, \ldots, \mathbf{q}_{N-1}\right) .
\end{aligned}
$$

This implies it commutes with the free representation of the Poincare group on $L^{2}\left(d q_{1} d q_{2}\right)$. One would therefore be inclined to make the change of variables (4.3)(4.5) once more for $\mathbf{q}_{1}, \mathbf{q}_{2}$ and then to add the interaction $V_{12}$ as in Section 4. However, this is only guaranteed to lead to an $S$-operator commuting with the latter representation if the factor $E_{q_{N}}$ in $H_{r}$ commutes with $V_{12}$. Thus, since $V_{12}$ commutes with $\mathbf{k}(12) \equiv\left(\mathbf{q}_{1}+\mathbf{q}_{2}\right) / M(12)$ by construction, it would commute with $M(12)$ and hence with $E_{\mathrm{q}_{1}}+E_{\mathrm{q}_{2}}=k_{0}(12) M(12)$. But then the $S$-operator does not exist!

It is possible to obtain an $S$-operator of the desired type as follows. Transform to variables $\mathbf{k}(12)$ and $\mathbf{q}(12)$ as above and then set $\mathbf{p}(12)=\mathbf{k}(12) M(12)=\mathbf{q}_{1}+\mathbf{q}_{2}$ (this is essentially the Bakamjian-Thomas transformation [32]). One then has

$$
E_{q_{1}}+E_{q_{2}}=\left(|\mathbf{p}(12)|^{2}+\left[\left(|\mathbf{q}(12)|^{2}+m_{1}^{2}\right)^{1 / 2}+\left(|\mathbf{q}(12)|^{2}+m_{2}^{2}\right)^{1 / 2}\right]^{2}\right)
$$

Now note that the factor $M(12)$ in the square brackets is just like $H_{v}{ }^{0}$ from Section 4, so that if we set $M(12) \rightarrow M(12)+V$ as in Section 4 we will get (for suitable $V$ ) an $S$-operator of the form (4.18) on $L^{2}(d \mathbf{p}(12) d \mathbf{q}(12))$ by the invariance principle for the wave operators. Since it commutes with $M(12)$, it will take the form (5.32) upon transforming back to $L^{2}\left(d \mathbf{k} d \mathbf{q}_{1} \cdots d \mathbf{q}_{N-1}\right)$, which is what we wanted. However, it is clear that if the other pair interactions are introduced in a similar way, the analysis of the resulting reduced Hamiltonian will be very complicated. (Note in particular that, e.g., the $1 N$ interaction should be defined by first transforming $\mathbf{q}_{1}, \mathbf{q}_{2}, \ldots$, $\mathbf{q}_{N-1} \rightarrow \mathbf{q}_{1},-\sum_{i=1}^{N-1} \mathbf{q}_{i}, \ldots, \mathbf{q}_{N-1}$, introducing interaction as above, and then transforming back!)

Summarizing, it seems to us that this scheme permits no analog of nonrelativistic pair potentials that leads to a completely satisfactory scattering theory. To prove (or disprove) this in a more cogent fashion would be an interesting problem in mathematical physics. (In this connection we mention that in Ref. [35] it has been argued that one can introduce interaction in a 3-particle system à la Bakamjian-Thomas and obtain wave operators having the cluster property. However, the proof given there seems inconclusive to us.)

\subsection{Coulomb Interaction for Arbitrary Spin}

Let us now consider the situation that some or all of the particles have nonzero spin. In this case one could still define the interaction by using (5.24) and (5.25) for 
$\mathbf{k}=0$. This means that all the $G^{s_{i}}$ equal the identity, while the $\mathbf{k}$ dependence of $V$ is governed by (5.19). Thus, the interaction would be spin independent in the center-ofmass frame. We shall now discuss an interaction that is more physical in this respect. It consists in taking again $V=\sum V_{i j}$, but now

$$
\begin{aligned}
\left(V_{12}(\mathbf{0}) g\right)_{\mathbf{a}}\left(\mathbf{q}_{1}, \mathbf{q}_{2}, \ldots, \mathbf{q}_{N-1}\right)= & \int d \mathbf{q}^{\prime} \hat{\phi}_{\mathbf{1 2}}\left(\frac{1}{2}\left(\mathbf{q}_{1}-\mathbf{q}_{2}\right)-\mathbf{q}^{\prime}\right) G^{s_{1}}\left(q_{1}, q_{1}^{\prime}\right)_{\alpha_{1} \beta_{1}} G^{s_{2}}\left(q_{2}, q_{2}^{\prime}\right)_{\alpha_{2} \beta_{2}} \\
& \times g_{\beta}\left(\frac{1}{2}\left(\mathbf{q}_{1}+\mathbf{q}_{2}\right)+\mathbf{q}^{\prime}, \frac{1}{2}\left(\mathbf{q}_{1}+\mathbf{q}_{2}\right)-\mathbf{q}^{\prime}, \ldots, \mathbf{q}_{N-1}\right),
\end{aligned}
$$

where

$$
\underset{2}{\mathbf{q}_{1}^{\prime}} \equiv \frac{1}{2}\left(\mathbf{q}_{1}+\mathbf{q}_{2}\right) \pm \mathbf{q}^{\prime},
$$

while the other $V_{i j}(\mathbf{0})$ are defined analogously. Here,

$$
G^{\mathrm{s}}\left(q, q^{\prime}\right) \equiv \mathrm{l}\left[M^{\mathrm{s}}(q) M^{\mathrm{s}}\left(q^{\prime}\right)+M^{\mathrm{s}}\left(I_{r} q\right) M^{\mathrm{s}}\left(I_{r} q^{\prime}\right)\right]
$$

where $I_{r} q \equiv\left(E_{q},-q\right)$ and where $M^{s}$ is the matrix multiplication operator

$$
M^{s}(q) \equiv\left(\frac{m}{E_{q}}\right)^{s} \mathscr{D}^{s}\left[\left(\frac{q}{m}\right)\right]
$$

Note that the $G^{s_{i}}$ are self-adjoint and that they satisfy (5.20) by virtue of (A8). Hence we can define $V_{\mathbf{1 2}}(\mathbf{k})$ (and likewise the other $V_{i j}(\mathbf{k})$ ) by replacing the $G^{s_{i}}\left(q_{i}, q_{i}^{\prime}\right)$ in (5.34) by the $\mathscr{G}^{s_{i}}\left(k, q_{i}, q_{i}^{\prime}\right)$ as defined in (5.19). Then the resulting operator $V$ commutes with $U_{f}(\Lambda)$, reduces to (5.24)-(5.26) if all $s_{i}$ are zero, and is again of the form (5.18), but now one needs $L \leqslant \frac{1}{2} N(N-1)$ terms in general (we have suppressed the index $l$ on $G_{l}^{s}$ for obvious reasons). Moreover, since the eigenvalues of $k$ are $\left(k_{\mathbf{0}}+|\mathbf{k}|\right)^{+1 / 2}$, one has

$$
\left\|M^{s}\right\|=2^{s} \text {. }
$$

It easily follows from this that $H_{r}(\mathbf{0})$ is positive if the potentials $\phi_{i j}$ are positive or "small." Since $H_{r}(\mathbf{k})$ is unitarily equivalent to $H_{r}(\mathbf{0})$ by $(5.19)$, we conclude that $H$ is positive in this case.

The choice (5.34), combined with Coulomb pair potentials, i.e.,

$$
\hat{\phi}(\mathbf{q})=-\left.\frac{\lambda}{2 \pi^{2}} ! \mathbf{q}\right|^{-2}, \quad \lambda \in R
$$

or equivalently

$$
\phi(\mathbf{x}) \equiv \equiv d \mathbf{q} \exp (i \mathbf{q} \cdot \mathbf{x}) \hat{\phi}(\mathbf{q})=-\lambda|\mathbf{x}|^{-1}
$$


is"of special interest, since it seems to us a natural generalization of the nonrelativistic $N$-body Coulomb interaction to the relativistic arbitrary spin framework considered here. Our arguments for this can be found in this and other subsections.

First, let us consider the case $N=-=2$ and $m_{2} \gg m_{1}$. We may then approximate $H_{r}(\mathbf{0})-m_{2}$ by the operator

$$
H^{s}(\lambda) \equiv E_{p}-\lambda V^{s}
$$

where $E_{p}$ is the operator of multiplication by $\left(|\mathbf{p}|^{2}+m^{2}\right)^{1 / 2}$ and where

$$
\left(V^{s} f\right)_{\alpha}(\mathbf{p})=\frac{1}{2 \pi^{2}} \int d \mathbf{p}^{\prime}\left|\mathbf{p}-\mathbf{p}^{\prime}\right|^{-2} G^{s}\left(p, p^{\prime}\right)_{\alpha \alpha^{\prime}} f_{\alpha^{\prime}}\left(\mathbf{p}^{\prime}\right)
$$

We have suppressed the spin index of particle 2 (since it is not acted on in this approximation) and have omitted the index on $m_{1}$ and $s_{1}$. Moreover, to emphasize that this is really an external field approximation we have changed $\mathbf{q}$ to $\mathbf{p}$. Thus, we may regard $H^{s}$ as an operator on $L^{2}(d \mathbf{p})^{2 s+1}$.

We shall now argue that in the present positive energy framework $H^{s}$ is a natural candidate to describe a light spin-s particle of charge $e$ in the field of a nucleus of charge $-Z e$ (note that we take $e<0$ ). In this case $\lambda=Z \alpha$, where $\alpha$ is the finestructure constant. First and foremost, it is plausible that $H^{\mathbf{1 / 2}}$ (which we shall presently define more precisely) has a bound-state spectrum that gives a rather more accurate description of the experimental data on hydrogen-like energy levels than the nonrelativistic Coulomb spectrum, since it presumably deviates only in order $\lambda^{6}$ from the spectrum of the Dirac operator (cf. e.g., Ref. [50])

$$
H_{D}(\lambda) \equiv-i \alpha \cdot \nabla+\beta m-\lambda|\mathbf{x}|^{-1}, \quad \lambda>0
$$

on $L^{2}(d \mathbf{x})^{4}$. One way to see this is to write

$$
\left(H^{1 / 2} f\right)(\mathbf{p})=\left(m+\frac{|\mathbf{p}|^{2}}{2 m}\right) f(\mathbf{p})-\frac{\lambda}{2 \pi^{2}} \int d \mathbf{p}^{\prime}\left|\mathbf{p}-\mathbf{p}^{\prime}\right|^{-2} f\left(\mathbf{p}^{\prime}\right)+(F f)(\mathbf{p}),
$$

and to regard $F$ as a fine-structure correction to the nonrelativistic Hamiltonian, which is the sum of a multiplicative "Sommerfeld correction"

$$
S(\mathbf{p})=\left(|\mathbf{p}|^{2}+m^{2}\right)^{1 / 2}-m-\frac{|\mathbf{p}|^{2}}{2 m}
$$

and a remaining correction

$$
(R(\lambda, m) f)(\mathbf{p})=\frac{\lambda}{2 \pi^{2}} \int d \mathbf{p}^{\prime}\left|\mathbf{p}-\mathbf{p}^{\prime}\right|^{-2}\left(1-G^{1 / 2}\left(p, p^{\prime}\right)\right) f\left(\mathbf{p}^{\prime}\right) .
$$


Then the first-order shift of the nonrelativistic level $m\left(1-\frac{1}{2} \lambda^{2} / n^{2}\right)$ with normalized wavefunction $\lambda^{3 / 2} \psi_{n l m s}(\lambda \mathbf{x})\left(s= \pm \frac{1}{2}\right)$ is the function

$$
C(\lambda)=C_{s}(\lambda)+C_{R}(\lambda)
$$

where

$$
\begin{aligned}
& C_{S}(\lambda)=\int d \mathbf{p}\left|\hat{\psi}_{n l m s}(\mathbf{p})\right|^{2} S(\lambda \mathbf{p}), \\
& C_{R}(\lambda)=\lambda^{2}\left(\hat{\psi}_{n l m s}, R(1, m / \lambda) \hat{\psi}_{n l m s}\right),
\end{aligned}
$$

and

$$
\hat{\psi}(\mathbf{p}) \equiv(2 \pi)^{-3 / 2} \int d \mathbf{x} \exp (-i \mathbf{p} \cdot \mathbf{x}) \psi(\mathbf{x})
$$

But one has, using (A7),

$1-G^{1 / 2}\left(p, p^{\prime} ; m / \lambda\right)=\lambda^{2}\left(-\frac{i}{4 m^{2}} \sigma \cdot \mathbf{p} \times \mathbf{p}^{\prime}+\frac{1}{8 m^{2}}\left|\mathbf{p}-\mathbf{p}^{\prime}\right|^{2}\right)+O\left(\lambda^{4}\right)$.

From this one readily infers that the $O\left(\lambda^{4}\right)$ correction consists of the usual Sommerfeld correction, spin-orbit term, and Darwin term occuring in the Pauli equation for the large components of the Dirac wavefunction. It is known (cf. Ref. [51 pp. 58-61 and 84]) that these terms give rise to first-order shifts to levels that coincide with the Dirac levels in order $\lambda^{4}$. Since the remaining corrections due to higher-order terms and higherorder perturbation theory are $O\left(\lambda^{6}\right)$, it follows that for $\lambda>0$ the discrete spectrum of $H$ coincides with that of $H_{D}$ up to $O\left(\lambda^{6}\right)$. (Of course, these arguments do not constitute a rigorous proof since we have not shown that (i) the energy levels of $H^{1 / 2}$ are analytic in $\lambda^{2}$ near zero like the Dirac levels, (ii) Rayleigh-Schrödinger theory makes sense for the perturbation $F$, (iii) $C_{S}$ and $C_{R}$ are analytic in $\lambda^{2}$ near zero.) Another way of seeing this will be discussed presently. However it is convenient to study first the operator $H^{s}$ for general $s$.

\subsection{Spectral Properties of $H^{s}$}

Qualitatively the spectrum of $H^{s}$ may be largely determined in a rigorous fashion by using recent results of Herbst [52] concerning the operator $\left(-\Delta+m^{2}\right)^{1 / 2}-$ $\lambda|\mathbf{x}|^{-1}$ on $L^{2}(d \mathbf{x})$, which is the Fourier transform of our $H^{0}(\lambda)$. Indeed, since the largest eigenvalue of the matrix $k_{0}^{-2 s}\left(\mathscr{Q}^{s}[\tilde{k}]+\mathscr{Q}^{s}[k]\right)$ is bounded above by $2^{2 s}$ for $s \geqslant \frac{1}{2}$, the relation

$$
\left\|E_{p}^{-1 / 2} V^{0} E_{p}^{-1 / 2}\right\|=\frac{1}{2} \pi
$$

(which is Eq. (2.19) in Ref. [52] in our notation) is easily seen to imply that

$$
\left\|E_{p}^{-1 / 2} V^{s} E_{p}^{-1 / 2}\right\| \leqslant 2^{2 s-2} \pi, \quad s \geqslant \frac{1}{2} .
$$


(This bound may be improved presumably.) Thus, if

$$
\lambda<2^{2-2 s} / \pi
$$

(which will be assumed from now on), we can define $H^{s}(\lambda)$ as the unique operator associated with the form sum of $E_{p}$ and $-\lambda V^{s}$. From now on $H^{s}$ will denote this operator. Note that $Q\left(H^{s}\right)=Q\left(E_{p}\right)$ since $Q\left(V^{s}\right) \supset Q\left(E_{p}\right)$ by (5.53), and that $H^{s}(\lambda)$ may differ from the Friedrichs extension of $E_{p}-\lambda V^{s} \uparrow D\left(E_{p}\right)$ if $\lambda \leqslant-2^{2-2 *} / \pi$. It then follows as in Ref. [52] that

$$
H^{s}(\lambda) \geqslant m\left[1-\left(2^{2 s-2} \pi \lambda\right)^{2}\right]^{1 / 2}, \quad s \geqslant \frac{1}{2},
$$

and that the essential spectrum of $H^{s}$ is $[m, \infty)$, so that the spectrum of $H^{s}$ in $(0, m)$ consists of finitely degenerate eigenvalues without accumulation point in $[0, m)$. (Of course, $H^{s}(\lambda) \geqslant m$ if $\lambda$ is negative.)

It does not immediately follow from Herbst's results that eigenvalues $\geqslant m$ and singular continuous spectrum are absent for $s>0$, since his virial theorem does not hold unmodified in this case, while $V^{s}$ does not scale like $V^{0}$. However, one can still show absence of singular continuous spectrum for $|\lambda|<2^{2-2 s} / \pi$ by appropriately combining his results with the dilation analyticity technique introduced in Ref. [53] and summarized in Ref. [37]. The proof briefly runs as follows. Define the scaling operator

$$
(u(\theta) f)(\mathbf{p})=e^{-3 \theta / 2} f\left(e^{-\theta} \mathbf{p}\right), \quad \theta \in R .
$$

Then one has, using (A7),

$$
u(\theta) M^{s}(m) u(\theta)^{*}=M^{s}\left(e^{\theta} m\right) .
$$

From the fact that the right-hand side extends to a pointwise analytic matrix multiplication operator in the strip $|\operatorname{Im} \theta|<\frac{1}{2} \pi$ whose pointwise $\theta$ derivatives are bounded at infinity (in p) it is not hard to see that the right-hand side in fact extends to an analytic bounded operator-valued function in $|\operatorname{Im} \theta|<\frac{1}{2} \pi$. Since $\left|\boldsymbol{M}^{s}\left(e^{\theta} m\right)\right|$ is a continuous function of $\operatorname{lm} \theta$ one infers from (5.53), fixing $\lambda_{0}$ with $\left|\lambda_{0}\right|<2^{2-2 s} / \pi$, that there exist $\delta, \epsilon>0$ such that

$$
\left|\lambda_{\mathbf{0}}\right|\left\|E_{p}^{-1 / 2} V^{s}\left(e^{i y} m\right) E_{p}^{-1 / 2}\right\| \leqslant 1-\delta
$$

for all $y$ with $-\epsilon \leqslant y \leqslant \epsilon$. Here, $V^{s}\left(e^{i y} m\right)$ is defined as in (5.42), but with $M^{s}(m)$ replaced by $M^{s}\left(e^{i y} m\right)$. Using this it can be shown that the form sum of the multiplication operator $H_{0}^{\prime}(\theta) \equiv\left(|\mathbf{p}|+e^{2 \theta} m^{2}\right)^{1 / 2}$ and $-\lambda_{0} V^{s}\left(e^{\theta} m\right)$ defines a strictly $m$-sectorial closed form $h_{s}^{\prime}(\theta)$ on $Q\left(E_{p}\right)$ for $|\operatorname{Im} \theta| \leqslant \bar{\epsilon}$, provided $0<\bar{\epsilon} \leqslant \min \left(\epsilon, \delta, \frac{1}{4} \pi\right)$. Clearly, $h_{s}^{\prime}(\theta)(\psi, \psi)$ is analytic in $|\operatorname{Im} \theta|<\frac{1}{2} \pi$ for $\psi \in Q\left(E_{p}\right)$, so that the associated operator family $H_{s}^{\prime}(\theta)$ is an analytic family of type $B$ in $|\operatorname{Im} \theta| \leqslant \bar{\epsilon}$ (cf. Refs. [37, 53]). It now follows as in Rer. [52] that the essential spectra of $H_{0}^{\prime}(\theta)$ and $H_{s}^{\prime}(\theta)$ coincide. (There is a misprint in the proof of Lemma 2.6, which contains the relevant ideas to show this:

$$
\left.\left\|(1-\chi)\left(1-e^{-\delta x^{2}}\right)\right\| \rightarrow 0 \quad \text { should read } \quad\left\|\left.! V\right|^{1 / 2}(1-\chi)\left(1-e^{-\delta x^{2}}\right)\right\| \rightarrow 0 \text {. }\right)
$$


Clearly, the operators $H_{0}(\theta) \equiv e^{-\theta} H_{0}^{\prime}(\theta)$ and $H_{s}(\theta) \equiv e^{-\theta} H_{s}^{\prime}(\theta)$ have analogous properties. An inspection of the proof of Theorem XIII 36 in Ref. [37] and of the essential spectrum of $H_{0}(\theta)$ now shows that the singular continuous spectrum of $H^{s}\left(\lambda_{0}\right)=H_{s}(0)$ is empty, as claimed.

We should like to complete our analysis of the operator $H^{8}$ by proving it has an infinite number of eigenvalues (counting multiplicity) smaller than $m$ if $\lambda>0$ (and $<2^{2-2 \times} / \pi$ by our standing assumption (5.54)). We first observe that

$$
u(\theta) H^{s}(m) u(\theta)^{*}=e^{-\theta} H^{s}\left(e^{\theta} m\right),
$$

which holds in the sense of quadratic forms on $Q\left(E_{p}\right)=Q\left(H^{s}\right)$. It is easy to see that this implies $H^{*}(1)$ has an eigenvalue $E$ if and only if $H^{s}(m)$ has an eigenvalue $m E$, the corresponding eigenfunctions being related by $\psi(m)=u(\ln m) \psi(1)$. Thus we can take $m=1$. Our claim is quite easy to prove for $s=0$ in view of the inequality

$$
H^{0}(\lambda)-1 \equiv\left(|\mathbf{p}|^{2}+1\right)^{1 / 2}-\lambda V^{0}-1 \leqslant H_{\mathrm{nr}}^{0}(\lambda) \equiv \frac{1}{2}|\mathbf{p}|^{2}-\lambda V^{0},
$$

which holds on $Q\left(H_{\mathrm{nr}}^{0}\right)=D\left(E_{p}\right) \subset Q\left(H^{0}\right)$ in the form sense. Since $H_{\mathrm{nr}}^{0}$ has an infinite number of negative eigenvalues

$$
-\frac{1}{2} \lambda^{2}=E_{1, \mathrm{nr}}<-\frac{1}{8} \lambda^{2}=E_{2, \mathrm{nr}}=\cdots=E_{5, \mathrm{nr}}<\cdots,
$$

an argument based on the min-max principle shows not only $H^{0}$ has an infinite number of eigenvalues $E_{1}{ }^{0} \leqslant E_{2}{ }^{0} \leqslant \cdots<1$, but also that

$$
E_{i}^{0} \leqslant E_{i, \mathrm{nr}}+1, \quad i=1,2, \ldots .
$$

(It seems plausible to us that the inequality

$$
H^{\mathrm{s}}-1 \leqslant H_{\mathrm{nr}}^{0}
$$

holds true for any $s, H_{\mathrm{nr}}^{0}$ being defined on $L^{2}(d \mathbf{p})^{2 s+1}$ in the obvious way. This would imply

$$
E_{i}{ }^{s} \leqslant E_{i, \mathrm{nr}}+1, \quad i=1,2, \ldots,
$$

where now of course the nonrelativistic level $\mathrm{nlm}$ is $2 s+1$-fold degenerate, so that $E_{i, \mathrm{nr}}$ in (5.64) $\leqslant E_{i, \mathrm{nr}}$ in (5.62). However, we have not proved this, so that (5.63) and 5.64) should be regarded as conjectures.)

To prove our assertion for general $s$ we first note that

$$
H^{s}(\lambda)-1 \leqslant H_{\mathrm{nr}}^{\mathrm{s}}(\lambda) \equiv \frac{1}{2}|\mathbf{p}|^{2}-\lambda V^{s}
$$

in the form sense on $D\left(E_{p}\right)$, which equals the form domain of the operator $H_{\mathrm{nr}}^{s}(\lambda)$ since $V^{s}$ is infinitesimally small w.r.t. $|\mathbf{p}|^{2}$. It is therefore sufficient to show that $H_{\mathbf{n r}}^{s}$ has infinitely many negative eigenvalues. Since the eigenvalues are monotone decreasing as $\lambda$ increases we only need to show that for any given $N$ we can find a $\lambda_{0}$ such that $H_{\mathrm{nI}}^{s}(\lambda)$ has $N$ negative eigenvalues for $0<\lambda \leqslant \lambda_{0}$. By the Rayleigh-Ritz principle 
this will follow if we succeed in finding orthonormal functions $\psi_{i}(\lambda) \in D\left(|\mathbf{p}|^{2}\right)$, $i=1, \ldots, N$, such that the matrix

$$
M_{i j}(\lambda) \equiv \lambda^{-2}\left(\psi_{i}(\lambda), H_{\mathrm{nr}}^{s}(\lambda) \psi_{j}(\lambda)\right), \quad i, j=1, \ldots, N
$$

is strictly negative definite for $0<\lambda \leqslant \lambda_{0}$. We take for the $\psi_{i}(\lambda)$ the eigenfunctions of the operator $H_{\mathrm{nr}}^{0}(\lambda)$ on $L^{2}(d \mathbf{p})^{2 s+1}$, corresponding to the $N$ "lowest" levels $n I m \alpha$ $(\alpha=1, \ldots, 2 s+1)$. From the scaling relations

$$
u(\theta) H_{\mathrm{nr}}^{0}(\lambda) u(\theta)^{*}=e^{-2 \theta} H_{\mathrm{nr}}^{0}\left(e^{\theta} \lambda\right)
$$

and

$$
u(\theta) V^{s}(1) u(\theta)^{*}=e^{-\theta} V^{s}\left(e^{\theta}\right)
$$

(which hold on $D\left(|\mathbf{p}|^{2}\right)$ in the operator sense) it then follows by taking $\theta=-\ln \lambda$ that

$$
\begin{aligned}
M_{i j}(\lambda) & \equiv \lambda^{-2}\left(\psi_{i}(\lambda),\left[\frac{1}{2}|\mathbf{p}|^{2}-\lambda V^{s}(1)\right] \psi_{j}(\lambda)\right) \\
& =-\delta_{i j} \frac{1}{2 n(i)^{2}}+\lambda^{-1}\left(\psi_{i}(\lambda),\left[V^{\mathbf{0}}-V^{s}(1)\right] \psi_{j}(\lambda)\right) \\
& =-\delta_{i j} \frac{1}{2 n(i)^{2}}+\left(\psi_{i}(1),\left[V^{0}-V^{s}(1 / \lambda)\right] \psi_{j}(1)\right)
\end{aligned}
$$

By dominated convergence the second term on the right-hand side goes to zero for $\lambda \rightarrow 0$. Consequently we can find a $\lambda_{0}$ such that $M_{i j}(\lambda)$ is strictly negative definite for $0<\lambda \leqslant \lambda_{n}$, which completes the proof of our assertion.

\subsection{The Relation between $H^{1 / 2}$ and the Dirac Operator}

Let us now come back to the relation of $H^{1 / 2}$ with the Dirac operator (5.43), as promised. By using the well-known spinor solutions to the free Dirac equation one can define a unitary opcrator $W: L^{2}(d \mathbf{p})^{4} \rightarrow L^{2}(d \mathbf{x})^{4}$ such that

$$
W^{-1} H_{D}(\lambda) W=\left(\begin{array}{cc}
E_{p} & 0 \\
0 & -E_{p}
\end{array}\right)-\lambda\left(\begin{array}{cc}
V_{++} & V_{+-} \\
V_{-+} & V_{--}
\end{array}\right)
$$

where the entries are $2 \times 2$ matrices acting on $L^{2}(d \mathbf{p})^{2}$. If one chooses $W$ as in Ref. [55], one concludes that

$$
E_{p}-\lambda V_{++}=H^{1 / 2}(\lambda)
$$

while

$$
-E_{p}-\lambda V_{--}=-\zeta H^{1 / 2}(-\lambda) \zeta^{*}
$$


where $\zeta$ is the $2 \times 2$ matrix $\left(\begin{array}{ccc}0 & 1 \\ -1 & 0\end{array}\right)$. Hence, (5.70) implies that

$W\left(\begin{array}{cc}H^{1 / 2}(\lambda) & 0 \\ 0 & -\zeta H^{1 / 2}(-\lambda) \zeta^{*}\end{array}\right) W^{-1}=P_{+}{ }^{0} H_{0}(\lambda) P_{+}{ }^{0}+P_{-}{ }^{0} H_{D}(\lambda) P_{-}{ }^{0} \equiv H_{D}^{\mathrm{appr}}(\lambda)$

where $P_{ \pm}{ }^{0}$ are the spectral projections of the free Dirac operator on the intervals $[m, \infty)$ resp. $(-\infty,-m]$, given by the well-known expressions $\frac{1}{2} \pm \frac{1}{2} E_{p}^{-1}(\alpha \cdot \mathbf{p}+\beta m)$ after Fourier transformation. But in view of our results on the spectrum of $H^{1 / 2}$ this relation implies that $H^{1 / 2}(\lambda)$ and $H_{D}^{\text {appr }}(\lambda)$ have the same discrete spectrum for $\lambda>0$. Let us therefore write

$$
H_{D}^{\mathrm{appr}} \equiv H_{D}+C
$$

and consider the level shift due to $C$ in perturbation theory. To evaluate the dominant term in the first-order shift we may expand the coefficients and exponents occurring in the Dirac wavefunctions (cf. Ref. [51] pp. 63-71, and references given there]) in $\lambda=Z \alpha$, retaining only the lowest order in $\lambda$. Note that the resulting $S$-wavefunctions are no longer singular at the origin. To find the expectation of $C$ for these wavefunctions we observe that

$$
\begin{aligned}
P_{+}{ }^{0}(\mathbf{p}) & P_{-}^{0}\left(\mathbf{p}^{\prime}\right)+P_{-}^{0}(\mathbf{p}) P_{+}\left(\mathbf{p}^{\prime}\right) \\
\quad= & \left(\frac{1}{2 m} \gamma \cdot\left(\mathbf{p}-\mathbf{p}^{\prime}\right)-\frac{i}{2 m^{2}} \mathbf{\Sigma} \cdot \mathbf{p} \times \mathbf{p}^{\prime}+\frac{1}{4 m^{2}}\left|\mathbf{p}-\mathbf{p}^{\prime}\right|^{2}\right)\left(1+O\left(m^{-2}\right)\right),
\end{aligned}
$$

where

$$
\boldsymbol{\Sigma} \equiv\left(\begin{array}{ll}
\sigma & 0 \\
0 & \sigma
\end{array}\right)
$$

(we use the Dirac representation of the $\gamma$-algebra). Hence one has

$$
C=\lambda\left[\frac{i}{2 m} \frac{\gamma \cdot \mathbf{r}}{r^{3}}+\frac{1}{2 m^{2} r^{3}} \mathbf{\Sigma} \cdot \mathbf{L}+\frac{\pi}{m^{2}} \delta(\mathbf{x})\right]+C_{r},
$$

where (formally) the term $C_{r}$ gives a contribution to the shift that is on the order of $\lambda^{2}$ times the contribution due to the first three terms. At first sight the result(5.77) seems to contradict our earlier conclusion, since the three terms clearly give rise to $O\left(\lambda^{4}\right)$ shifts. (Remember that $\gamma$ is off-diagonal so that it only couples the $O(1)$ and $O(\lambda)$ components.) However, we have checked explicitly that in this order the term containing $\gamma$ cancels the "Darwin term" for the $1 S_{1 / 2}$ and $2 S_{1 / 2}$ states, while it cancels the "spin-orbit" term for the $2 P_{1 / 2}$ and $2 P_{3 / 2}$ states. More generally, the fact that the $O\left(\lambda^{4}\right)$ shift is zero can presumably be shown by appropriately using the formal relation

$$
\frac{1}{2 m^{2}}\left[H_{D},\left[H_{D}, \frac{\lambda}{r}\right]\right]=C-C_{r} .
$$


Thus, if these perturbation theory arguments can be trusted we come once more to the conclusion that the discrete spectrum of $H^{1 / 2}\left(Z_{\alpha}\right)$ will be very close to that of $H_{D}(Z \alpha)$ for low $Z$. It would be interesting to determine how the energy levels deviate from those of $I I_{D}$ in order $\lambda^{6}$, and to establish whether applying first-order perturbation theory to (5.74) leads to the same result as applying it to (5.44).

\subsection{Physical Aspects of $H^{s}\left(s \neq \frac{1}{2}\right)$ and Reduced Hamiltonians}

For $s \geqslant 1$ the operator $H^{s}$ cannot be compared with Lorentz covariant wave equations as we did in the spin-1/2 case, since higher-spin equations cannot be cast in the form of theories with a self-adjoint Hamiltonian as soon as interaction is present. The choice (5.42) was made since it seems to be a natural generalization of $H^{1 / 2}$ to higher-spin values that can be shown to have "reasonable" spectral properties. It would be of interest to calculate the $O\left(\lambda^{4}\right)$ fine structure in perturbation theory like in the spin- $1 / 2$ case, using the relation

$$
\begin{aligned}
G^{s}\left(p, p^{\prime}\right)= & 1+\frac{1}{2 m^{2}}\left[\mathbf{S} \cdot\left(\mathbf{p}+\mathbf{p}^{\prime}\right)\right]^{2} \\
& +\frac{i}{2 m^{2}} \mathbf{S} \cdot \mathbf{p} \times \mathbf{p}^{\prime}-\frac{s}{2 m^{2}}\left(|\mathbf{p}|^{2}+\left|\mathbf{p}^{\prime}\right|^{2}\right)+O\left(m^{-4}\right)
\end{aligned}
$$

(cf. (A.22)). This would give a model for levels of spin-1 mesons or the $\Omega^{-}$-particle in the field of a nucleus. However, as far as we know such exotic atoms have not been observed.

For $s-0$ the situation is different since $\pi$-mesic and $K$-mesic energy levels have been observed (cf. e.g., Ref. [56 and references given there]). As in the case of $H^{1 / 2}$ and the Dirac operator, the $O\left(\lambda^{4}\right)$ fine structure predicted by $H^{0}(\lambda)$ (in perturbation theory) coincides with the $O\left(\lambda^{4}\right)$ fine structure found via the customary procedure of solving the Klein-Grordon equation with the Coulomb potential (cf. Eq. (42.21) in Ref. [57] and Eq. (13.9) in Ref. [51]). However, deviations do occur in $O\left(\lambda^{6}\right)$. For instance, one can explicitly evaluate the first-order "Sommerfeld correction" (5.48) for the nonrelativistic ground state. It reads

$$
C_{S}(\lambda)=m\left[\left(1-2 \lambda^{2}\right)\left(1-\lambda^{2}\right)^{-5 / 2}-1-\frac{1}{2} \lambda^{2}\right],
$$

so that the first-order $O\left(\lambda^{6}\right)$ coefficient is $-35 / 16$. Since one obtains $-21 / 16$ as the coefficient of the Klein-Gordon ground-state energy

$$
E_{1}(\lambda)=m\left[\frac{1}{2}+\frac{1}{2}\left(1-4 \lambda^{2}\right)^{1 / 2}\right]^{1 / 2}
$$

and the $O\left(\lambda^{6}\right)$ shift due to second-order perturbation theory is negative, the levels cannot coincide in $O\left(\lambda^{6}\right)$. However, it seems rather unlikely that such deviations can be observed, since radiative corrections are already on the order of $\lambda^{5} \ln \lambda$, and, moreover, the strong interaction and the finite size of the nucleus cannot be neglected in mesonium [56]. Assuming perturbation theory is a reliable guide one may therefore take the point 
of view (as in the Dirac casc) that $H^{0}(\lambda)$ is just as "good" a model of low- $Z$ mesonic energy levels as the Klein-Gordon equation. Moreover (in contrast to the Dirac case) there is no fixed self-adjoint Hamiltonian that represents the energy of the meson in the usual treatment, so that the resulting energy levels are outside the bounds of the conventional interpretation of quantum mechanics. From this point of view the operator $H^{0}(\lambda)$, which obviously does not suffer from this shortcoming and moreover has no negative energy spectrum, could be even considered as a better model. It would be of interest to determine the discrete spectrum of $H^{0}(\lambda)$ more accurately and in particular to establish whether observable differences with the Klein-Gordon levels result for larger $Z$, where perturbation theory certainly should be distrusted. Of course, it is very unlikely one could solve the eigenvalue problem explicitly, but the lowest levels might be determined numerically by using suitable trial wavefunctions. However, we do not know whether sufficient accuracy can be achieved to make a sensible comparison with the Klein-Gordon levels possible.

Until now we have dealt with the approximate Hamiltonian $H^{s}$ that arises by taking the limit $m_{2} \rightarrow \infty$ in the exact reduced Hamiltonian

$$
\begin{aligned}
\left(H_{r}(\mathbf{0}) g\right)_{\alpha \beta}(\mathbf{q})= & \left(E_{\alpha_{1}}+E_{q_{3}}\right) f_{\alpha \beta}(\mathbf{q})-\frac{\lambda}{2 \pi^{2}} \int d \mathbf{q}^{\prime}\left|\mathbf{q}-\mathbf{q}^{\prime}\right|^{-2} \\
& \times G^{s_{1}}\left(q_{1}, q_{\mathbf{1}}^{\prime}\right)_{\alpha \alpha^{\prime}} G^{s_{2}}\left(q_{2}, q_{2}^{\prime}\right)_{\beta \beta^{\prime}} g_{\alpha^{\prime} \beta^{\prime}}\left(\mathbf{q}^{\prime}\right) .
\end{aligned}
$$

As mentioned before, this amounts to an external field approximation and as such clearly breaks the Lorentz invariance of the theory. However, we should like to emphasize that the operator (5.82) provides a fully relativistic description of a twobody system. Indeed, the rigorous definition and analysis of this operator can proceed along similar lines as that of $H^{s}$. In particular, it is positive for negative $\lambda$ and for small positive $\lambda$, its essential spectrum equals $\left[m_{1}+m_{2}, \infty\right)$, and it has infinitely many eigenvalues $<m_{1}+m_{2}$ for $\lambda>0$. As far as we know a similar Lorentz invariant (as opposed to Lorentz covariant) description is not possible in the framework of the usual wave equations.

Let us now comment on the physical merits of the operator (5.82) and its obvious pair potential generalization to the $N$-body case. If we consider a hydrogen-like atom we have $\lambda=Z \alpha, m_{1}=m_{e}$, and $m_{2}=m_{N} \gg m_{e}$. In this case the discrete spectrum of $I_{r}(0)$ will presumably deviate only very slightly from that of $H^{1 / 2}(Z \alpha)$ (apart from the nuclear rest mass shift). Indeed, in perturbation theory the main effect is the replacement of the electron mass by the reduced mass; the "fine-structure" terms (5.79) due to the nucleus are suppressed by a relative factor $\left(m_{e} / m_{N}\right)^{2}$. In view of this and the arguments presented above we expect that the bound-state levels of these reduced Hamiltonians give a rather more accurate description of experimental reality than their nonrelativistic counterparts. Thus, for atomic systems these operators may be regarded as fully relativistic positive energy approximations to the "ultimate" positive energy relativistic quantum field theory whose resonances presumably describe the observed atomic spectra. 
It is an obvious question to ask whether this approximation could be improved by choosing other reduced Hamiltonians than the simple and symmetric choice $(5.82)$. We feel that this is an issue that would merit a thorough investigation. Of course, it is extremely unlikely that one can define reduced Hamiltonians whose energy levels agree with experiment to the remarkable degree of accuracy one obtains in the customary treatment based on the Dirac equation or Schrödinger equation together with the Breit operator and radiative corrections (cf. Refs. [51, 58]). However, if one uses these corrections as a guide, one may be able to introduce well-defined operators that give an even more accurate description of atoms and two-body systems like positronium and muonium than provided by (5.82) and its $N$-body analogs. As an illustrative example we shall consider the hyperfine structure of hydrogen-like energy levels in the next subsection.

\subsection{Hyperfine Structure}

In view of (5.79) the lowest-order spin-spin coupling term in (5.82) gives a groundstate energy splitting on the order of $\lambda^{6} / m_{N}^{2}$ (formally, since the relevant integral diverges). As is well-known, the actual splitting is on the order of $\lambda^{4} / m_{N}$. An inspection of the $1 / m$ expansion of $M^{s}$ (cf. (5.37) and the Appendix) shows that a splitting of the correct sign and order of magnitude may be achieved by making the replacement

$$
\begin{aligned}
G^{s_{1}}\left(q_{1}, q_{1}^{\prime}\right) G^{s_{2}}\left(q_{2}, q_{2}^{\prime}\right) \rightarrow & \frac{1}{2}\left[M^{s_{1}}\left(q_{1}\right) M^{s_{1}}\left(q_{1}^{\prime}\right) M^{s_{2}}\left(q_{2}\right) M^{s_{2}}\left(q_{2}^{\prime}\right)\right. \\
& \left.+M^{s_{1}}\left(I_{r} q_{1}\right) M^{s_{1}}\left(I_{r} q_{1}^{\prime}\right) M^{s_{2}}\left(I_{r} q_{2}\right) M^{s_{2}}\left(I_{r} q_{2}^{\prime}\right)\right]
\end{aligned}
$$

in (5.82). Taking the nonrelativistic ground-state wavefunction for reduced mass $\mu=m_{1} m_{2} /\left(m_{1}+m_{2}\right)$ one finds that the ground-state splitting is determined by the operator

$$
C_{\mathrm{hf}}^{\mathbf{1}}=\frac{8}{3} \frac{\mu^{3}}{m_{1} m_{2}}(Z \alpha)^{4}\left(\mathbf{S}_{1} \cdot \mathbf{S}_{2}\right)
$$

in spin space. This coincides with the Fermi formula $[50,51,59]$ but for a factor $g_{N} / 2 Z$. A moment's thought shows that this factor can be taken into account by replacing $M^{s_{2}}\left(p_{2}\right)$ in (5.83) by $M^{s_{2}}\left(p_{2}\right)^{g_{N} / 2 Z}$. The resulting operator is well-defined and gives the correct lowest-order hyperfine structure for the ground state (and of course, as before, the correct lowest-order fine structure). However, this is unfortunately not the case for the $n S$-states with $n>1$, since a calculation shows that their splitting is determined by the operator

$$
C_{\mathrm{hf}}^{n}=\frac{2}{3} \frac{\mu^{3}}{m_{1} m_{2}} g_{N} Z^{3} \alpha^{4}\left(\frac{3}{n^{3}}-\frac{1}{n^{4}}\right)\left(S_{1} \cdot S_{2}\right)
$$

while the factor in brackets equals $2 / n^{3}$ in the Fermi formula. Thus for $n=2$ the splitting is already $25 \%$ too large. 
Although it does not seem possible to obtain the correct lowest-order $S$-level hyperfine structure by modifying $H_{r}(\mathbf{0})$ as above, one can achieve this by adding the operator

$$
\begin{aligned}
\left(T_{\mathrm{hr}}(\mathbf{0}) g\right)(\mathbf{q})= & \frac{\alpha g_{N}}{2 \pi^{2}} \int d \mathbf{q}^{\prime}\left|\mathbf{q}-\mathbf{q}^{\prime}\right|^{-2}\left[\mathbf{S}_{1} \cdot\left(\mathbf{q}-\mathbf{q}^{\prime}\right)\right]\left[\mathbf{S}_{2} \cdot\left(\mathbf{q}-\mathbf{q}^{\prime}\right)\right] \\
& \cdot \frac{m_{1} m_{2}}{E_{q_{1}} E_{q_{1}^{\prime}} E_{q_{2}} E_{q_{2}^{\prime}}} g\left(\mathbf{q}^{\prime}\right)
\end{aligned}
$$

to (5.82) (with $\lambda=Z \alpha$ ). Indeed, it is easy to see that for $S$-states this term leads to the Fermi formula in lowest order. The operator $T_{\mathrm{hf}}(0)$ is again of the form (5.18) with $\mathbf{k}=0$ and $L=1$. The corresponding $G^{s}$ satisfy (5.20) by virtue of (A23) and therefore $T_{\mathrm{hP}}$, defined via (5.19), commutes with $U_{f}(\Lambda)$. It also follows as before that the new $H_{r}(\mathbf{0})$ is positive and has infinitely many bound states below its essential spectrum $\left[m_{1}+m_{2}, \infty\right)$ as long as $Z$ is low and/or $s_{1}$ and $s_{2}$ are not too large (given that $\alpha \simeq 1 / 137$ and that $g_{N}=O(1)$; note that the values of $m_{1}$ and $m_{2}$ do not play a role).

\subsection{Coupling of a Quantized Radiation Field for $s=\frac{1}{2}$}

There is one last issue that we should like to consider bricfly, viz., how onc could couple a quantized radiation field to the Hamiltonians studied above. Our discussion is formal and tentative, and will be restricted to the spin-1/2 case. Let us first consider $H^{1 / 2}(Z \alpha)$. At first sight it would seem a good choice to add an interaction term

$$
\begin{aligned}
(A f)_{\alpha}(\mathbf{p})= & -\frac{e}{2} \int d \mathbf{p}^{\prime}\left(-\frac{m}{E_{p}}\right)^{1 / 2}\left[\left(\frac{p}{m}\right)_{\wedge} \sigma^{k}\left(\frac{p^{\prime}}{m}\right)_{\wedge}-\left(\frac{p}{m}\right)^{\wedge} \sigma^{k}\left(\frac{p^{\prime}}{m}\right)^{\wedge}\right]_{\alpha \alpha^{\prime}}\left(-\frac{m}{E_{p^{\prime}}}\right)^{1 / 2} \\
& \cdot \hat{\Lambda}^{k}\left(\mathbf{p} \quad \mathbf{p}^{\prime}\right) f_{\alpha^{\prime}} \cdot\left(\mathbf{p}^{\prime}\right),
\end{aligned}
$$

and to subsequently quantize the magnetic field $\mathbf{A}(\mathbf{x})$ in the usual way, since $E_{p}+A$ is essentially $P_{+}{ }^{0} H_{D}^{\prime} P_{+}{ }^{0}$, where

$$
H_{D^{\prime}}=-i \alpha \cdot \nabla+\beta m-e \boldsymbol{\nabla} \cdot \mathbf{A}(\mathbf{x}) .
$$

Thus, $E_{p}+A$ would be related to $H_{D}^{\prime}$ in the same way that $H^{1 / 2}$ is related to $H_{D}$ (cf. (5.70) and (5.71)). However, this coupling would violently disagree with the observed Thompson scattering cross section. Indeed, as is well-known (cf. Ref. [60]) this cross section is dominated by negative energy intermediate states, which we have excluded by construction. Another way to see that (5.87) is not a "good" proposal is to expand it in powers of $1 / \mathrm{m}$ :

$$
\begin{aligned}
(A f)_{\alpha}(\mathbf{p})= & -\frac{e}{2 m} \int d \mathbf{p}^{\prime}\left[\left(p^{k}+p^{\prime k}\right) \delta_{\alpha \alpha^{\prime}}+i \epsilon_{i j k} \sigma_{\alpha \alpha^{\prime}}^{i}\left(p^{j}-p^{\prime j}\right)\right] \\
& \cdot \hat{A}^{k}\left(\mathbf{p}-\mathbf{p}^{\prime}\right) f_{\alpha^{\prime}}\left(\mathbf{p}^{\prime}\right)+O\left(m^{-3}\right) .
\end{aligned}
$$


This agrees with the Pauli equation, except that the usual $|\mathbf{A}|^{2}$ term is missing. But this term is responsible for diamagnetism, and in quantized form for the Thompson cross section [60].

We conclude from this that one should add a term quadratic in $\mathbf{A}$ to (5.87) to improve agreement with experiment. However, we do not venture an opinion on what the best choice would be. It is also not clear to us how one should couple $\mathbf{A}$ in the fully relativistic case. The most obvious possibility would be to add to $H$ a term (5.87) for each momentum $\mathbf{p}_{i}$, and then to quantize $\mathbf{A}$, but one could also add such terms to $H_{r}(\mathbf{0})$, replacing the momenta $\mathbf{p}_{i}$ by the center-of-mass momenta $\mathbf{q}_{i}$. The latter choice would certainly be more convenient in calculating transition probabilities. Another issue of interest would be to establish whether one can calculate radiative corrections to the bound-state levels without invoking positrons, i.e., just using a coupling like (5.87). However, this may be quite hard, since there is no reason to expect that the relevant integrals will be convergent, and, in contrast to the case of quantum electrodynamics, it does not seem possible to renormalize these integrals by employing Lorentz and gauge invariance. Indeed, the absence of negative energy states makes it unlikely one can cast the integrals into a formally Lorentz and gauge invariant form. This may be seen already by regarding (5.87) as an external field coupling: If one combines (5.87) with a coupling to an external electric field as in $H^{1 / 2}$, it is far from clear that a Lorentz covariant and gauge invariant $S$-operator results (this is presumably not the case, except for the Born term), properties that are known to hold formally in the Dirac case, and that can in fact be rigorously proved for external fields that are test functions on space-time [55, 61].

\section{APPENDIX}

In this Appendix we collect some well-known facts concerning the (restricted) Lorentz and Poincaré groups in three space dimensions. It is included both to fix our notation and for reasons of completeness. For more information and proofs we refer to Refs. $[19,62,63]$.

The restricted Lorentz group is the identity component of $S O(1,3)$, i.e., the set of all real-valued $4 \times 4$ matrices $A$ that satisfy

$$
\Lambda x \cdot \Lambda y=x \cdot y, \quad \forall x, y \in R^{4}
$$

and that can be continuously connected to the identity matrix. In (AI) $p \cdot q$ denotes the Lorentz inner product

$$
p \cdot q=p_{0} q_{0}-\mathbf{p} \cdot \mathbf{q} \cdot
$$

Any such $A$ may be uniquely decomposed as

$$
A=R B
$$


where $R$ is a rotation (i.e., $(R x)_{0}=x_{0}$ and det $R=1$ ) and $B$ is a pure boost (i.e., symmetric). Note this is just the polar decomposition of $\Lambda$, so that $B=\left(\Lambda^{*} \Lambda\right)^{1 / 2}$.

Let $k=\left(k_{0}, \mathbf{k}\right)$ be a unit 4-vector, i.e., $k \cdot k=1$. Then the matrix

$$
\underline{k} \equiv k_{\mathbf{0}}+\mathbf{k} \cdot \boldsymbol{\sigma}=\left(\begin{array}{ll}
k^{0}+k^{3} & k^{1}-i k^{2} \\
k^{1}+i k^{2} & k^{0}-k^{3}
\end{array}\right)
$$

is Hermitean and has determinant 1. Conversely, any such matrix may be written in the form (A4) and therefore uniquely determines a unit 4-vector. The inverse of $k$ is clearly

$$
\tilde{k}=k_{0}-\mathbf{k} \cdot \boldsymbol{\sigma} .
$$

From now on we restrict ourselves to unit 4-vectors with $k_{\mathbf{0}}>0$. Then $k$ and $\tilde{k}$ are positive, so we can introduce their square roots:

$$
\underset{\alpha}{k}=(k)^{1 / 2}, \quad \hat{k}=(\tilde{k})^{1 / 2} .
$$

It is easy to verify that $k$ is explicitly given by

$$
k=\left[2\left(k_{0}+1\right)\right]^{-1 / 2}(1+\underset{s}{k})
$$

Let $A$ be any $2 \times 2$ matrix with complex entries and determinant 1, i.e., let $A \in S L(2, C)$. It can be shown that $S L(2, C)$ and the restricted Lorentz group are homomorphic, the homomorphism $\Lambda(A)$ being such that

$$
A \underset{\sim}{k} A^{*}=(\Lambda(A) k)_{\sim}
$$

or, equivalently,

$$
A^{*-1} \tilde{k} A^{-1}=(\Lambda(A) k)^{\sim}
$$

Moreover, if $A(A)=A(B)$, then $A= \pm B$, and if $U|A|$ is the polar decomposition of $A$, then $\Lambda(U) \Lambda(|A|)$ is the polar decomposition (A3) of $\Lambda(A)$. Thus, $S U(2)$ corresponds to rotations, while Hermitean $A$ correspond to pure boosts.

Let $B(k)$ denote the pure boost

$$
B(k):(1, \mathbf{0}) \rightarrow\left(k_{0}, \mathbf{k}\right)
$$

Explicitly,

$$
B(k)=\left(\begin{array}{cc}
k^{0} & k^{j} \\
k^{i} & \delta_{i j}+k^{i} k^{j} /\left(1+k^{0}\right)
\end{array}\right), \quad i, j=1,2,3 .
$$

Then we conclude by setting $k=(1,0)$ and $A=k$ in (A8) that

$$
A(k)=B(k)
$$


Note also that

$$
B(k) I_{r}=I_{r} B(k)^{-1},
$$

where

$$
I_{r} x \equiv\left(x_{0},-\mathbf{x}\right)
$$

We now introduce the matrix

$$
U(k, A) \equiv\left(\Lambda(A)^{-1} k\right)^{\wedge} A^{-1} k .
$$

Using (A8) it readily follows that $U(k, A)$ is unitary. One also verifies that

$$
U(k, A)^{*}=U\left(\Lambda(A)^{-1} k, A^{-1}\right) .
$$

Denoting by $R(k, \Lambda)$ the rotation corresponding to $U(k, A)$ we then have, using (A12),

$$
R(k, A)=B\left(\Lambda^{-1} k\right)^{-1} \Lambda^{-1} B(k)
$$

resp.

$$
R(k, \Lambda)^{-1}=R\left(\Lambda^{-1} k, \Lambda^{-1}\right) .
$$

Note that (A8) implies that

$$
U(k, A)=A^{*}, \quad \forall A \in S U(2),
$$

from which

$$
R(k, \Lambda)=\Lambda^{-1}
$$

if $A$ is a rotation. It is also illuminating to observe that $R(k, \Lambda)$ equals the identity if $A$ is a pure boost in the direction $\mathbf{k}$ or $-\mathbf{k}$. Indeed, in this case the corresponding $A$ is easily seen to commute with $\tilde{k}$ so that $\left(\Lambda(A)^{-1} k\right)^{\wedge}=\hat{k} A$ by (A9).

One can represent $S L(2, C)$ on the $N$-fold symmetric tensor product of $C^{2}$ with itself (denoted $T_{N}$ ) by the map $A \rightarrow \otimes_{N} A$. We shall denote this representation (known to be irreducible) by $\mathscr{D}^{N / 2}[A]$. Taking the orthonormal base $e_{+} \equiv\left(\begin{array}{l}1 \\ 0\end{array}\right)$, $e_{-} \equiv\left(\begin{array}{l}0 \\ 1\end{array}\right)$ for $C^{2}$ one gets an orthonormal base for $T_{N}$ by setting

$$
e_{\alpha}=\operatorname{Sym}\left[\left(\otimes_{\alpha-1}^{\otimes} e_{-}\right) \otimes\left(\underset{N+1-\alpha}{\otimes} e_{+}\right)\right] / C_{\alpha}, \quad \alpha=1, \ldots, N+1,
$$

where $C_{\alpha}$ is a normalization factor. We shall denote the generator of rotations on $T_{N}$ by $\mathrm{S}$. Explicitly, one has

$$
\mathbf{S}=\sum_{i=1}^{2 s} 1 \otimes \cdots \otimes \frac{1}{2} \boldsymbol{\sigma} \otimes \cdots \otimes 1,
$$


where $s \equiv N / 2$. Note that (A8) implies that

$$
\mathscr{D}^{s}[A] \mathbf{S} \mathscr{D}^{s}\left[A^{*}\right]=\Lambda(A)^{-1} \mathbf{S}, \quad \forall A \in S U(2) .
$$

Finally, if $M$ is an operator on $T_{N}$ we define

$$
M_{\alpha \beta}=\left(e_{\alpha}, M e_{\beta}\right), \quad \alpha, \beta=1, \ldots, N+1 .
$$

The restricted Poincaré group is the set of pairs $(a, \Lambda)$ (where $a$ is a 4-vector and $\Lambda$ belongs to the restricted Lorentz group) with the multiplication

$$
\left(a_{1}, \Lambda_{1}\right)\left(a_{2}, \Lambda_{2}\right)=\left(a_{1}+\Lambda_{1} a_{2}, \Lambda_{1} \Lambda_{2}\right) .
$$

In the main text we use its irreducible representations $(m, s)$ where $m>0$ and $s \geqslant 0$ is half an integer. These representations describe free particles of mass $m$ and spin $s$, and may be defined on $L^{2}\left(R^{3}, d \mathbf{p}\right)^{2 s+1}$ by setting

$$
\left(U_{f}(a, \Lambda) f\right)_{\alpha}(\mathbf{p})=\exp (i p \cdot a)\left(\frac{\left(\Lambda^{-1} p\right)_{0}}{p_{0}}\right)^{1 / 2} U^{s}\left[\frac{p}{m}, A\right]_{\alpha \beta}^{*} f_{\beta}\left(\overrightarrow{\Lambda^{-1} p}\right) .
$$

Here, $A$ is such that $\Lambda(A)=\Lambda$ and

$$
U^{s}[k, A] \equiv \mathscr{D}^{s}[U(k, A)] .
$$

Moreover,

$$
p=\left(E_{p}, \mathbf{p}\right)
$$

where

$$
E_{p} \equiv\left(|\mathbf{p}|^{2}+m^{2}\right)^{1 / 2}
$$

It is straightforward to verify that $U(a, \Lambda)$ is a unitary representation. Note that the representation is two-valued if $s$ is a half-integer. However, it may be regarded as a true representation of the universal covering group, $i S L(2, C)$. Observe also that the Lorentz boosts $U_{f}(0, B(k))$ are time-independent operators, corresponding to the Heisenberg picture of quantum mechanics, which is employed throughout this paper.

\section{ACKNOWLEDGMENTS}

We wish to thank J. Mather, E. Seiler, B. Simon, and A. S. Wightman for valuable discussions. Part of this work was done at the Aspen Center for Physics during the summer of 1978. We should like to thank the Center for its hospitality.

Note added in proof. The author recently came across a paper by Mutze [66], where under quite general conditious lack of clustering is shown to occur in the Bakamjian-Thomas framework. From it, we learned about the extensive work of Sokolov on relativistic particle dynamics: His work can be traced from the recent reference [67]. He has studied in particular a point form dynamics as considered in this paper, its relation to front and instant form dynamics, and the cluster problem discussed in Section 5.2. 


\section{REFERENCES}

1. P. Federbush, Phys. Rev. 121 (1961), 1247-1249.

2. A.S. Wightman, Introduction to some aspects of the relativistic dynamics of quantized fields, in "High Energy Electromagnetic Interactions and Field Theory"(M. Lévy, Ed.), pp. 171-289, Gordon \& Breach, New York, 1967.

3. B. Schroer, T. T. Truong, and P. Weisz, Ann. Phys. (N.Y.) 102 (1976), 156-169.

4. M. SATO, T. MrWA, AND M. JIMBO, Field theory of the 2-dimensional Ising model in the scaling limit, RIMS preprint 207, 1976.

5. A. B. Zamolodchikov, Comm. Math. Phys. 55 (1977), 183-186.

6. A. B. Zamolodchikov aNd A. B. Zamolodchikov, Nucl. Phys. B 133 (1978), 525-535.

7. M. KARowsKI, Exact S-matrices and form factors in $1+1$ dimensional field theoretic models with soliton behavior, in "Winter Workshop," Les Houches, 1978.

8. A. C. ScotT, F. Y. F. ChU, and D. W. McLaughein, Proc. IEEE 61 (1973), 1443-1483.

9. R. HrRotA, J. Phys. Soc. Japan 33 (1972), 1459-1463.

10. L. A. Takhtadzhyan, Soviet Physics JETP 39 (1974), 228-233.

11. E. A. Kuznetsov And A. V. Mikhailov, Theor. Math. Phys. 30 (1977), 193-200.

12. J. B. MCGuIRE, J. Mathematical Phys. 5 (1964), 622-636.

13. E. Brézin ANd J. Zinn-Justin, C.R. Acad. Sci. Paris 263 (1966), 670-673.

14. C. N. YANG, Phys. Rev. 168 (1968), 1920-1923.

15. V. E. Zakharov and A. B. Shabat, Soviet Physics JETP 34 (1972), 62-69.

16. V. E. ZakHarov and A. B. Shabat, Soviet Physics JETP 37 (1973), 823-828.

17. F. A. Berezin and V. N. Sushko, Soviet Physics JETP 21 (1965). 865-873.

18. H. Bergknory and H. B. Thacker, Phys. Rev. Lett. 42 (1979), 135-138.

19. R. F. Streater AND A. S. Wightman, "PCT, Spin and Statistics and All That," Benjamin, New York, 1964.

20. D. Ruelle, Helv. Phys. Acta 35 (1962), 147 163.

21. R. Jost, "The General Theory of Quantized Fields," Amer. Math. Soc., Providence, R.I., 1965.

22. M. Reed AND B. Simon, "Methods of Modern Mathematical Physics. III. Scattering Theory," Academic Press, New York, 1979.

23. J. FröHLICH, Comm. Math. Phys. 47 (1976), 269-310.

24. J. FRöHLICH, Quantum sine-Gordon equation and quantum solitons in two space-time dimensions, in "Renormalization Theory" (G. Velo and A. S. WightMAN, Eds.), pp. 371-415, Reidel, Dordrecht, 1976.

25. "Constructive Quantum Field Theory" (G. Velo and A. S. Wightman, Eds.), Lecture notes in Physics 25, Springer, Berlin/New York, 1973.

26. B. Simon, "The $P(\phi)_{2}$ Euclidean (quantum) Field Theory," Princeton Univ. Press, Princeton, N.J., 1974.

27. H. Airault, H. P. McKean, And J. Moser, Comm. Pure Appl. Math. 30 (1977), 95-148.

28. D. V. Choodnovsky ANd G. V. Choodnovsky, Nucl. Phys. B 40 (1973), 339-352.

29. P. HÄHLEN, Nucl. Phys. B 102 (1976), 67-92.

30. P. A. M. DiraC, Rev. Mod. Phys. 21 (1949), 392-399.

31. L. H. ThOMas, Phys. Rev. 85 (1952), 868-872.

32. B. Bakamitan and L. H. Thomas, Phys. Rev. 92 (1953), 1300-1310.

33. L. L. Foldy, Phys. Rev. 122 (1961), 275-288.

34. R. Fong AND J. SUCHer, J. Mathematical Phys. 5 (1964), 456-470.

35. F. Coester, Helv. Phys. Acta 38 (1965), 7-23.

36. M. Pauri and G. M. Prosperi, J. Mathematical Phys. 17 (1976), 1468-1495.

37. M. ReEd AND B. Simon, "Methods of Modern Mathematical Physics. IV. Analysis of Operators," Academic Press, New York, 1978.

38. M. ReEd ANd B. Simon, "Methods of Modern Mathematical Physics. I. Functional Analysis," Academic Press, New York, 1972.

39. J. FröHLICH, Comm. Math. Phys. 54 (1977), 135-150. 
40. W. Thirring, "Lehrbuch der mathematischen Physik. 1. Klassische dynamische Systeme," Springer, Berlin/New York/Wien, 1977.

41. R. Abraham AND J. E. Marsden, "Foundations of Mechanics," Benjamin/Cummings, Reading, Mass., 1978.

42. H. GoldsteIn, "Classical Mechanics," Addison-Wesley, Reading, Mass., 1950.

43. E. H. Wichmann and J. H. Crichton, Phys. Rev. 132 (1963), 2788-2799.

44. W. HunzIKer, J. Mathematical Phys. 6 (1965), 6-10.

45. K. HePr, Helv. Phys. Acta 37 (1964), 659-662.

46. M. ReED, B. Simon, "Methods of Modern Mathematical Physics. II. Fourier Analysis, SelfAdjointness," Academic Press, New York, 1975.

47. B. Srmon, Comm. Math. Phys. 23 (1971), 37-48.

48. E. T. Whittaker and G. N. Watson, "A Course of Modern Analysis," Cambridge Univ. Press, London/New York, 1973.

49. D. G. CURRIE AND T. F. JORDAN, Interactions in relativistic classical particle mechanics, in "Quantum Theory and Statistical Physics" (A. O. Barut and W. E. Brittin, Eds.), Lectures in Theoretical Physics XA, pp. 91-139, Gordon \& Breach, New York, 1968.

50. J. D. BJorken AND S. D. Drell, "Relativistic Quantum Mechanics," McGraw-Hill, New York, 1964.

51. H. A. Bethe and E. E. Salpeter, "Quantum Mechanics of One- and Two-Electron Atoms," Springer, Berlin/New York, 1957.

52. I. W. Herbst, Comm. Math. Phys. 53 (1977), 285-294.

53. J. Aguilar and J. M. Combes, Comm. Math. Phys. 22 (1971), 269-279.

54. T. KATo, "Perturbation Theory for Linear Operators," Springer, Berlin/New York, 1966.

55. S. N. M. RuiJsenaArs, J. Mathematical Phys. 18 (1977), 720-737.

56. "International School of Physics of Exotic Atoms (G. Fiorentini and G. Torelli, Eds.), Pisa, 1977.

57. L. I. Schiff, "Quantum Mechanics," McGraw-Hill, New York, 1968.

58. B. E. Lautrup, A. Peterman, and E. de Rafael, Phys, Rep. 3 (1972), 193-260.

59. E. Fermi, $Z$. Phys. 60 (1930), 320-333.

60. J. SakURAI, "Advanced Quantum Mechanics," Addison-Wesley, Reading, Mass., 1967.

61. S. N. M. RuJusenaARs, J. Functional Analysis 33 (1979), 47-57.

62. E. Wigner, Ann. of Math. 40 (1939), 149-204.

63. A. S. Wightman, L'invariance dans la mécanique quantique relativiste, in "Relations de dispersion et particules élémentaires" (C. de Witt, R. Omnes, Eds.), pp. 159-226, Hermann, Paris, 1960.

64. V. Enss, Comm. Math. Phys. 61 (1978), 285-291.

65. B. Simon, Duke Math. J. 46 (1979), 119-168.

66. U. Mutze, J. Math. Phys. 19 (1978), 231-236.

67. S. N. Sokolov AND A. N. Shatnil, Theor. Math. Phys. 37 (1979), 1029-1038. 